Dissertations No. 1906

\title{
Resolvent Estimates and Bounds on Eigenvalues for Schrödinger and Dirac Operators
}

\author{
Alexandra Enblom
}

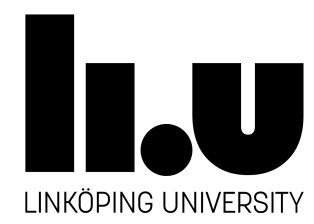

Department of Mathematics Linköping, 2018 
Linköping Studies in Science and Technology. Dissertations No. 1906

Resolvent Estimates and Bounds on Eigenvalues for Schrödinger and Dirac Operators

Copyright (C) Alexandra Enblom, 2018

Department of Mathematics

Linköping University

SE-581 83 Linköping, Sweden

Email: alexandra.enblom@liu.se

ISSN 0345-7524

ISBN 978-91-7685-362-7

Printed by LiU-Tryck, Linköping, Sweden, 2018 


\section{Abstract}

This thesis concerns the spectral theory of Schrödinger and Dirac operators. The main results relate to the problems of estimating perturbed eigenvalues. The thesis is based on four papers.

The first paper focuses on the problem of localization of perturbed eigenvalues for multidimensional Schrödinger operators. Bounds for eigenvalues, lying outside the essential spectrum, are obtained in terms of the Lebesgue's classes. The methods used make it possible to consider the general case of non-self-adjoint operators, and involve the weak Lebesgue's potentials. The results are extended to the case of the polyharmonic operators.

In the second paper, the problem of location of the discrete spectrum is solved for the class of Schrödinger operators considered on the half-line. The general case of complex-valued potentials, imposing various boundary conditions, typically Dirichlet and Neumann conditions, is considered. General mixed boundary conditions are also treated.

The third paper is devoted to Dirac operators. The case of spherically symmetric potentials is considered. Estimates for the eigenvalues are derived from the asymptotic behaviour of the resolvent of the free Dirac operator. For the massless Dirac operators, whose essential spectrum is the whole real line, optimal bounds for the imaginary part of the eigenvalues are established.

In the fourth paper, new Hardy-Carleman type inequalities for Dirac operators are proven. Concrete Carleman type inequalities, useful in applications, Agmon and also Treve type inequalities are derived from the general results by involving special weight functions. The results are extended to the case of the Dirac operator describing the relativistic particle in a potential magnetic field. 


\section{Populärvetenskaplig sammanfattning}

Huvudmålet för avhandlingen är att inom området matematisk fysik beskriva fenomen som påverkar de spektrala egenskaperna hos Schrödinger- och Diracoperatorer. Särskild uppmärksamhet ägnas åt problemet med att beskriva strukturen hos det såkallade spektrumet för sådana operatorer. Dessa problem är viktiga inom tillämpningar i spridningsteori (scattering theory), men även i spektralteori. För att uppnådetta, uppskattas de såkallade resolventerna hos de aktuella operatorerna, genom att beskriva deras beteende nära det kontinuerliga spektret. Sådan information är särskilt viktig främst pågrund av problem inom matematisk fysik, spridningsteori, kvantfysik och andra relaterade områden. För att fåmer exakt information om resolventernas egenskaper är det ofta lämpligt att använda Hardy- och Carlemanolikheter, eller andra liknande olikheter. För Schrödingeroperatorer finns en rik litteratur om sådana olikheter, men även om det finns gott om litteratur för Dirac-operatorer såär det området lite mindre utforskat. Av den anledningen fokuserar många av problem i avhandlingen påHardy-Carlemanolikheter för multidimenssionella Dirac-operatorer. Baserat påde beräkningar som utförs för resolventerna, erhålls information om placeringen av de stärda egenvärdena utanför det kontinuerliga spektret. Metoderna är till stor del baserade påmetoder för störningsteori för linjära operatorer. 


\section{Acknowledgments}

I would like to express my gratitude to my supervisor Ari Laptev. Thank you for introducing me to this interesting scientific topic, for all the useful discussions and for giving me such an opportunity, as to work in Linköping University. It was also an adventure to be a part of your journey and meet you in various places. I would also want to thank Vladimir Kozlov for always being near and helping me with everything I had problems with.

Department of Mathematics at LIU is a very nice place to work at and therefore I would like to thank all the colleagues, the administration and all the $\mathrm{PhD}$ students for making it more enjoyable.

Finally, I would like to thank my parents, my dad for teaching me to joke, my mom for being my best friend and my sister for always being there for me. My biggest appreciation goes to my husband Andreas and my two children Alexander and William. You give me strength to go on. 


\section{Contents}

Abstract ...................... i i

Populärvetenskaplig sammanfattning . . . . . . . . . . . . . iii

Acknowledgments . . . . . . . . . . . . . . . . . v v

Contents . . . . . . . . . . . . . . . . . . . vii

$1 \quad$ Introduction and Summary 1

1.1 Setting of the problems $\ldots \ldots \ldots \ldots \ldots \ldots$

1.2 Factorization scheme . . . . . . . . . . . . . . . . . 2

1.3 Analytic Fredholm Theorem . . . . . . . . . . . . . . . . 7

2 Discussion of Paper I $\quad 11$

2.1 Overview of Paper I . . . . . . . . . . . . . . . . 11

2.1.1 Schrödinger Operators . . . . . . . . . . . . . . . . . 11

2.1.2 Polyharmonic Operators . . . . . . . . . . . . . . . . . . . 15

2.1.3 Weak Lebesgue potentials . . . . . . . . . . . . . . 16

3 Discussion of Paper II $\quad 19$

3.1 Overview of Paper II . . . . . . . . . . . . . . . . . . . . 19

4 Discussion of Paper III 23

4.1 Overview of Paper III . . . . . . . . . . . . . . . 23

5 Discussion of Paper IV 29

5.1 Overview of Paper IV . . . . . . . . . . . . . . . . . 29

$\begin{array}{ll}\text { Paper I } & 41\end{array}$

$\begin{array}{ll}\text { Paper II } & 67\end{array}$

$\begin{array}{ll}\text { Paper III } & 81\end{array}$

$\begin{array}{ll}\text { Paper IV } & 101\end{array}$ 


\section{1 - Introduction and Summary}

\subsection{Setting of the problems}

This thesis is devoted to the spectral theory of Schrödinger and Dirac operators, which so far presents a fruitful research field to solve various problems in quantum mechanics, mathematics physics, mathematical analysis itself and other related domains (see [Gla66], [LS91], [RS78], [RS75], [RS79], [RS72], [BS87], [Tha92], [FH10], [LS10], [Sch81] and also the literatures cited there). One of the main problems is the investigation of the structure of the spectrum of the considered operator.

The first results in this regard, and then developed in various aspects, were based on H. Weyl's [Wey09] criterion concerning the invariance property of the essential spectrum under compact or, more generally relatively compact, perturbations. Weyl's criterion states that if $H_{0}$ is a self-adjoint operator acting in a Hilbert space, and $B$ is a relatively compact operator with respect to $H_{0}$, then the essential spectra of the operators

$$
H_{0} \quad \text { and } \quad H=H_{0}+B
$$

are the same. Under these conditions there can appear perturbed eigenvalues outside the essential spectrum. The set of those eigenvalues is no more than countable and its points of accumulation can only be on the essential spectrum. Moreover, each of such eigenvalues is of finite multiplicity. It is natural, but also important, to evaluate the perturbed eigenvalues, describe their asymptotic behaviour, determine the location regions of them, etc. These problems occur naturally in contexts of spectral theory having their significance especially viewing the requirements in mathematical physics and quantum mechanics. Namely on such problems the present thesis is focused. Bounds on the location of perturbed eigenvalues are obtained, in particular, for Schrödinger and Dirac type operators, by considering the general case of non-self-adjoint operators. The methods which we apply allow us to consider the operators on Lebesgue's classes $L_{p}(1<p<\infty)$. Moreover, even if the problem is set for operators considered on Hilbert spaces the evaluations are made involving Banach space technique. The results obtained here are mainly based on the operator-theoretical methods developed from the point of view of perturbation theory. In order to obtain as general and accurate results as possible, we will make extensive use of the technique of factoring the perturbation operators originated in [Sch60], used in [Pro64] (also in [SWW64]) and developed in [KK66], [Kur67] and [Kur73a] (see also [Kur73b]) from the point of view of the necessities in the scattering theory and spectral theory itself. In the next section, a version of the factorization scheme adopted to the situations which we consider in this work, is presented. 


\subsection{Factorization scheme}

In what follows, $\mathcal{X}, \mathcal{Y}, \ldots$ are assumed to be Banach spaces unless otherwise stated. The norms are denoted by $\|\cdot\|$ without designating the space, there will be no confusion.

A linear operator from $\mathcal{X}$ into $\mathcal{Y}$ is a linear mapping $T$ of a linear manifold of $\mathcal{X}$, called the domain of $T$ and denoted $D(T)$, into $\mathcal{Y}$. The set $R(T):=T D(T)$ of all $y \in \mathcal{Y}$ of the form $y=T x$ with $x \in D(T)$ is called the range (or image) of $T$. The null space (kernel) of $T$, i.e., the set all $x \in D(T)$ such that $T x=0$, will be denoted by $\mathcal{N}(T)$. If a linear manifold $D_{0}$ is contained in $D(T)$, one can consider the restriction of $T$ to $D_{0}$ to mean an operator, denoted by $T / D_{0}$, with domain $D_{0}$ acting by $T / D_{0} x=T x$ for $x \in D_{0}$. If $S$ and $T$ are linear operators from $X$ into $Y$, it said that $S$ is an extension of $T$ (or $T$ is a restriction of $S$ ) and write $T \subset S$ whenever $D(T) \subset D(S)$ and $S x=T x$ for all $x \in D(T)$.

The set of all closed densely defined linear operators acting from $\mathcal{X}$ into $\mathcal{Y}$ is denoted by $\mathcal{C}(\mathcal{X}, \mathcal{Y}) . \mathcal{B}(\mathcal{X}, \mathcal{Y})$ stands for the space of all bounded linear operators on $\mathcal{X}$ to $\mathcal{Y}$. We preserve the same notation $\|\cdot\|$ for the operator norm in $\mathcal{B}(\mathcal{X}, \mathcal{Y})$. Instead of $\mathcal{C}(\mathcal{X}, \mathcal{X})$ and $\mathcal{B}(\mathcal{X}, \mathcal{X})$ we write $\mathcal{C}(\mathcal{X})$ and $\mathcal{B}(\mathcal{X})$, respectively.

For any closed operator $T$ in $\mathcal{X}$, the resolvent set, the spectrum, and the point spectrum of $T$ are denoted by $\rho(T), \sigma(T)$ and $\sigma_{p}(T)$, respectively. The part of the discrete spectrum $\sigma_{d}(T)\left(\subset \sigma_{p}(T)\right)$ is defined as the set of all isolated eigenvalues of $T$ of finite multiplicities. The essential spectrum of $T$ is denoted by $\sigma_{e s s}(T)$. For brevity the resolvent of $T$ is denoted by $R(z ; T)$, that is, $R(z ; T):=(T-z I)^{-1}$, $z \in \rho(T)$. Recall that $R(z ; T)$ has domain $\mathcal{X}$ and range the domain $D(T)$ of $T$. If a densely defined operator $T$ is bounded, its (unique) extension on the whole space will be denoted by $[T]$.

Now, let $H_{0}$ be a closed densely defined operator on $\mathcal{X}$, and consider a perturbation given by the operator $V$. Our aim here is to describe an extension $H$ of $H_{0}+V$, suitable for our purposes, by means of the technique of factoring the perturbation, essentially, proposed by T.Kato in [Kat66]. In case $\mathcal{X}$ is a Hilbert space, $V$, and $H_{0}$ as well, is not necessary assumed to be symmetric or bounded. The basic assumptions are listed below. They are, to some extent, different from those in [Kat66], as well as, in [KK66], [Kur67] or [Kur73a], in which there is considered exclusively the case of Hilbert spaces. Furthermore, in mentioned works $H_{0}$ is always supposed to be self-adjoint.

Assumption 1.2.1. $V$ can be written formally as $V=A B$, where $A$ and $B$ are closed densely defined operators acting $B$ from $\mathcal{X}$ to another space $\mathcal{Y}$ and $A$ from $\mathcal{Y}$ to $\mathcal{X}$, respectively. Furthermore, for $z \in \rho\left(H_{0}\right)$ the operator $B R\left(z ; H_{0}\right)$ is bounded, that is, $B R\left(z ; H_{0}\right) \in \mathcal{B}(\mathcal{X}, \mathcal{Y})$ and the (densely defined) operator $R\left(z ; H_{0}\right) A$ has a (it is unique) bounded extension $\left[R\left(z ; H_{0}\right) A\right]$ on the whole space $\mathcal{Y}$.

Assumption 1.2.2. For one, or equivalently all, $z \in \rho\left(H_{0}\right)$ the densely defined operator $B R\left(z ; H_{0}\right) A$ has a bounded extension $\left[B R\left(z ; H_{0}\right) A\right]$ on the whole space $\mathcal{Y}$, let it be denoted by $Q(z)$, i.e.,

$$
Q(z):=\left[B R\left(z ; H_{0}\right) A\right], \quad z \in \rho\left(H_{0}\right) .
$$


Assumption 1.2.3. There exists a regular point $z$ of $H_{0}$ such that the operator $I+Q(z)$ (I denotes the identity operator) is invertible, with its inverse belonging to $\mathcal{B}(\mathcal{Y})$.

Remark 1.2.4. Under Assumptions 1.2 .1 and 1.2 .2 the range of $\left[R\left(z ; H_{0}\right) A\right]$ is contained in the domain $D(B)$ of $B$, i.e., the operator $B\left[R\left(z ; H_{0}\right) A\right]$ is well defined, and

$$
Q(z)=B\left[R\left(z ; H_{0}\right) A\right], \quad z \in \rho\left(H_{0}\right) .
$$

To show this fact, we let $v=\left[R\left(z ; H_{0}\right) A\right] u$ for any $u$ in $\mathcal{Y}$. Then there exists a sequence $\left(u_{n}\right)$ of elements $u_{n}$ in the domain $D(A)$ such that $u_{n} \rightarrow u$ in $\mathcal{Y}$ and $R\left(z ; H_{0}\right) A u_{n} \rightarrow v$ in $\mathcal{X}$. Since $Q(z)$ is bounded, it follows that and $B R\left(z ; H_{0}\right) A u_{n}=Q(z) u_{n} \rightarrow Q(z) u$, and so we have $R\left(z ; H_{0}\right) A u_{n} \rightarrow v$ in $\mathcal{X}$ and $B R\left(z ; H_{0}\right) A u_{n} \rightarrow Q(z) u$ in $\mathcal{Y}$. Since $B$ was supposed to be closed we get $v \in D(B)$ and $B v=Q(z) u$, and the claim is shown.

Remark 1.2.5. In case $\mathcal{X}$ is a Hilbert space, $H_{0}$ is self-adjoint (in $\mathcal{X}$ ) and if $A \in \mathcal{C}(\mathcal{Y}, \mathcal{X})$ and $B \in \mathcal{C}(\mathcal{X}, \mathcal{Y})$ such that $D\left(A^{*}\right) \supset D\left(H_{0}\right)$ and $D(B) \supset D\left(H_{0}\right)$, then the assumption 1.2.1 is fulfilled.

Indeed, the operators $B R\left(z ; H_{0}\right)$ and $A^{*} R\left(\bar{z} ; H_{0}\right)$ for $z \in \rho\left(H_{0}\right)$ are bounded by the closed graph theorem and, since $\left(A^{*} R\left(\bar{z} ; H_{0}\right)\right)^{*} \supset R\left(z ; H_{0}\right) A$ the (densely defined) operator $R\left(z ; H_{0}\right) A$ has a (unique) bounded extension $\left[R\left(z ; H_{0}\right) A\right]$ on $\mathcal{Y}$.

Under the assumptions 1.2.1, 1.2.2 and 1.2.3 one can define the operator-valued function

$$
R(z):=R\left(z ; H_{0}\right)-\left[R\left(z ; H_{0}\right) A\right](I+Q(z))^{-1} B R\left(z ; H_{0}\right)
$$

for all $z \in \rho\left(H_{0}\right)$ whenever $I+Q(z)$ is invertible as in the assumption 1.2.3. It turns out that thus defined operator-valued function $R(z)$ is the resolvent of a closed operator $H$ which is an extension of $H_{0}+V\left(=H_{0}+A B\right)$. This will be the definition of our perturbed operator.

In order to show that the operator-valued function $R(z)$ is a resolvent of an operator $H$ we proceed similarly as in [Kat66]. For we apply a known fact (see [HP74], Theorem 5.8.3) according with an operator-valued function defined on a subset $\Omega$ of the complex plane $\mathbb{C}$ is the resolvent of some closed linear operator $H$ if and only if $R(z)$ satisfies the first resolvent equation

$$
R\left(z_{1}\right)-R\left(z_{2}\right)=\left(z_{1}-z_{2}\right) R\left(z_{1}\right) R\left(z_{2}\right), \quad z_{1}, z_{2} \in \Omega
$$

and that $R(z)$ (for any $z \in \Omega$ ) has an inverse (not necessarily bounded). Under these conditions $H$ is expressed by

$$
H=z I+R(z)^{-1}
$$

that does not depend of $z, z \in \Omega$.

Next, we let $\Omega$ be the set of all $z \in \rho\left(H_{0}\right)$ such that the inverse $I+Q(z)$ exists as a bounded operator on $\mathcal{Y}$, i.e., the set on which $R(z)$ is defined.

Lemma 1.2.6. The null space of $R(z)$ for each $z \in \Omega$ is trivial. 
Proof. Multiplying (1.2) by $B$ from the left by $B$ and noting (1.1) we obtain

$$
\begin{gathered}
B R(z)=B R\left(z ; H_{0}\right)-B\left[R\left(z ; H_{0}\right) A\right](I+Q(z))^{-1} B R\left(z ; H_{0}\right)= \\
=(I+Q(z)-Q(z))(I+Q(z))^{-1} B R\left(z ; H_{0}\right)= \\
=(I+Q(z))^{-1} B R\left(z ; H_{0}\right),
\end{gathered}
$$

i.e.,

$$
B R(z)=(I+Q(z))^{-1} B R\left(z ; H_{0}\right), \quad z \in \Omega .
$$

Now, if $R(z) u=0$ for an element $u$ in $\mathcal{H}$, then $B R\left(z ; H_{0}\right) u=0$ and, also, $R\left(z ; H_{0}\right) u=0$ by $(1.2)$, so that $u=0$.

Next we note the following useful relations

$$
\begin{gathered}
{\left[R\left(z_{1} ; H_{0}\right) A\right]-\left[R\left(z_{2} ; H_{0}\right) A\right]=\left(z_{1}-z_{2}\right) R\left(z_{1} ; H_{0}\right)\left[R\left(z_{2} ; H_{0}\right) A\right],} \\
B R\left(z_{1} H_{0}\right)-B R\left(z_{2} ; H_{0}\right)=\left(z_{1}-z_{2}\right) B R\left(z_{1} ; H_{0}\right) R\left(z_{2} H_{0}\right),
\end{gathered}
$$

and, also,

$$
Q\left(z_{1}\right)-Q\left(z_{2}\right)=\left(z_{1}-z_{2}\right) B R\left(z_{1} ; H_{0}\right)\left[R\left(z_{2} H_{0}\right) A\right],
$$

which are simple consequences of the first resolvent equation for the unperturbed operator $H_{0}$ under conditions supposed by the assumptions 1.2.1 and 1.2.2.

Lemma 1.2.7. $R(z)$ satisfies the first resolvent equation (1.3).

Proof. Let $z_{1}, z_{2} \in \Omega$. Multiplying the expressions for $R\left(z_{1}\right)$ and $R\left(z_{2}\right)$ given by the formula (1.2) and then using the relations (1.5), (1.6) and (1.7), we get the desired equation (1.3) after simple calculations.

Thus, the operator-valued function defined by (1.2) is indeed a resolvent of a closed operator $H$, so that we can write

$$
R(z)=R(z ; H), \quad z \in \Omega .
$$

From (1.2) and (1.4) if follows that

$$
R(z ; H)-R\left(z ; H_{0}\right)=-\left[R\left(z ; H_{0}\right) A\right] B R(z ; H), \quad z \in \Omega .
$$

The relation (1.8) together with:

$$
R(z ; H)-R\left(z ; H_{0}\right)=-[R(z ; H) A] B R\left(z ; H_{0}\right), \quad z \in \Omega .
$$

can be regarded as the second resolvent equations.

Based on (1.8) or (1.9) it can easily be proven that $H$ is an extension of $H_{0}+A B$. For if we take an element $u \in D\left(H_{0}+A B\right)\left(=D\left(H_{0}\right) \cap D(A B)\right)$ and set $v=$ $\left(H_{0}-z I\right) u$ for any (fixed) $z \in \Omega$, then $u=R\left(z ; H_{0}\right) v$ and from (1.9), it follows that

$$
R(z ; H) v-u=-[R(z ; H) A] B u
$$


and, since $B u \in D(A)$ (u belongs to $D(A B)$ ), we get

$$
\begin{gathered}
u=R(z ; H) v+R(z ; H) A B u= \\
=R(z ; H)\left(\left(H_{0}-z I\right) u+A B u\right)= \\
=R(z ; H)\left(H_{0}+A B-z I\right) u .
\end{gathered}
$$

It is seen that $u$ belongs to the range of $R(z ; H)$, that is, to the domain of $H$, and, moreover,

$$
(H-z I) u=\left(H_{0}+A B-z I\right) u,
$$

or, which is the same,

$$
H u=\left(H_{0}+A B\right) u .
$$

By this we conclude that $H$ is indeed an extension of $H_{0}+A B$.

We have the following theorem.

Theorem 1.2.8. Let $H_{0}$ and $A, B$ be operators satisfying the assumptions 1.2.1, 1.2.2 and 1.2.3. Then, there exists a closed densely defined operator $H$ that is an extension of $H_{0}+A B$ with its resolvent expressed by the formula (1.2).

Remark 1.2.9. In [Kur73a] (see also [KK66] and [Kur67]) the scheme described above was considered by supposing additionally that the operator $H_{0}$ is selfadjoint, and $A, B$ factorizing the perturbation operator $V$ are closed satisfying

$$
\left\langle B u, A^{*} v\right\rangle=\left\langle A^{*} u, B v\right\rangle, \quad u, v \in D\left(A^{*}\right) \cap D(B),
$$

$(\langle\cdot, \cdot\rangle$ denotes the scalar product in the corresponding Hilbert space $\mathcal{X})$ and such that the operator $Q(z)$, the extension of $B R\left(z ; H_{0}\right) A$, is completely continuous for every $z \in \rho\left(H_{0}\right)$. Under these conditions the operator $H$ determined by (1.2) is self-adjoint [KK66] (see Theorem 1 [KK66]). Namely, such an extension is taken as the basic operator for the study from the scattering theory point of view (see [Kat66], [Kur67], [Kur73a], and the references cited there).

The discrete spectrum of $H$ can be described in terms of the operator-valued function $Q(z)$. Namely, we have the following.

Theorem 1.2.10. Suppose the assumptions 1.2.1, 1.2.2 and 1.2.3 are fulfilled, and let $H$ be determined by the formula (1.2). Then a point $\lambda, \lambda \in \rho\left(H_{0}\right)$, is an eigenvalue of $H$ if and only if -1 is an eigenvalue of $Q(\lambda)$. Moreover, the (geometric) multiplicity of $\lambda$ coincides with that of -1 as an eigenvalue of $Q(\lambda)$.

Proof. Let $\lambda \in \rho\left(H_{0}\right)$ be an eigenvalue of $H$, that is,

$$
H u=\lambda u, \quad u \neq 0 .
$$

Fix $z \in \rho\left(H_{0}\right) \cap \rho(H)$, and write (1.11) in the form

$$
u=(\lambda-z) R(z ; H) u \text {. }
$$

Therefore, in view of (1.2), we have

$$
u=(\lambda-z)\left(R\left(z ; H_{0}\right)-\left[R\left(z ; H_{0}\right) A\right](I+Q(z))^{-1} B R\left(z ; H_{0}\right)\right) u .
$$


Now, we denote

$$
v=(I+Q(z))^{-1} B R\left(z ; H_{0}\right) u,
$$

and multiply on both sides of (1.13) with $B R\left(z ; H_{0}\right)$, we then get

$$
B R\left(\lambda ; H_{0}\right) u=(\lambda-z)\left(B R\left(\lambda ; H_{0}\right) R\left(z ; H_{0}\right) u-B R\left(\lambda ; H_{0}\right)\left[R\left(z ; H_{0}\right) A\right] v\right),
$$

or, taking into account relations (1.6) and (1.7),

$$
B R\left(\lambda ; H_{0}\right) u=B R\left(\lambda ; H_{0}\right) u-B R\left(z ; H_{0}\right) u-(Q(\lambda)-Q(z)) v .
$$

Substituting in (1.15)

$$
B R\left(z ; H_{0}\right) u=(I+Q(z)) v
$$

we have

$$
(I+Q(z)) v-(Q(\lambda)-Q(z) v=0
$$

or

$$
Q(\lambda) v=-v
$$

Notice that $v \neq 0$, otherwise if $v=0$, then $B R\left(\lambda ; H_{0}\right) u=0$ by (1.16) that implies $\left(H_{0}-\lambda I\right) R\left(z ; H_{0}\right) u=0$ in virtue of (1.13). As was supposed $\lambda$ is a regular point for $H_{0}$, so $R\left(z ; H_{0}\right) u=0$, a contradiction.

Conversely, suppose (1.17) with $v \neq 0$. Again, we fix a point $z \in \rho\left(H_{0}\right) \cap \rho(H)$ and let

$$
u=\left[R\left(\lambda ; H_{0}\right) A\right] v
$$

We have

$$
\begin{aligned}
& (\lambda-z) R(z ; H) u=(\lambda-z)\left(R\left(z ; H_{0}\right)-\left[( R ( z ; H _ { 0 } ) A ] ( I + Q ( z ) ) ^ { - 1 } B \left(R\left(z ; H_{0}\right) u=\right.\right.\right. \\
= & (\lambda-z)\left(R\left(z ; H_{0}\right)\right)\left[R\left(\lambda ; H_{0}\right) A\right] v-\left[R\left(z ; H_{0}\right) A\right](I+Q(z))^{-1} B R\left(z ; H_{0}\right)\left[R\left(\lambda ; H_{0}\right) A\right] v,
\end{aligned}
$$

and, by (1.5) and (1.7), we can continue

$$
\begin{gathered}
(\lambda-z) R(z ; H) u=\left[R\left(\lambda ; H_{0}\right) A\right] v-\left[R\left(z ; H_{0}\right) A\right] v-\left[R\left(z ; H_{0}\right) A\right](I+Q(z))^{-1}(Q(\lambda)-Q(z)) v= \\
=u-\left[R\left(z ; H_{0}\right) A\right] v+\left[R\left(z ; H_{0}\right) A\right](I+Q(z))^{-1}(I+Q(z)) v= \\
=u-\left[R\left(z ; H_{0}\right) A\right] v+\left[R\left(z ; H_{0}\right) A\right] v=u
\end{gathered}
$$

i.e., (1.12), that is equivalent to (1.11). So $\lambda$ is an eigenvalue of $H$.

The second assertion follows from the fact that each of (1.14) and (1.18) determines a one-to-one mapping from the eigenspace of $H$ associated with $\lambda$ onto the corresponding one of $Q(\lambda)$.

Under the assumptions 1.2.1, 1.2.2 and 1.2.3, if it turns out that the values $Q(z), z \in \rho\left(H_{0}\right)$, are compact operators, the essential spectrum of $H$ coincides with that of the unperturbed operator $H_{0}$. This fact is explained in the next section via the analytic Fredholm theorem [Goh51] accommodated to the Banach spaces. 


\subsection{Analytic Fredholm Theorem}

The purpose of this section is to make further remarks on the spectrum of the perturbed operator $H$ constructed with the technique of factoring the perturbation. In the previous discussions we found that the discrete spectrum of the perturbed operator can be completely described in terms of the holomorphic operator-valued function

$$
Q(z)=B\left[R\left(z ; H_{0}\right) A\right], \quad z \in \rho\left(H_{0}\right) .
$$

Now we give additional conditions on the factors $A$ and $B$ involved in the factorization of the perturbation operator $V$ for $H_{0}$ and $H \sim H_{0}+V$ to have the same essential spectrum. For that will need the following additional assumption.

Assumption 1.3.1. The values $Q(z), z \in \rho\left(H_{0}\right)$ are compact operators.

Since we are dealing with Banach spaces, before proceeding to the main theorem, it is necessary to specify the appropriate terminology in order to avoid any misunderstanding.

Let $\mathcal{X}$ be a Banach space. An operator $T$ is said to be compact if $T$ maps bounded sets into relatively compact sets in $\mathcal{X}$. $A$ compact $T$ cannot have a bounded inverse, unless $\mathcal{X}$ is a finite-dimensional space, which means that $\lambda=0$ always belongs to the spectrum of $T$. Other points of the spectrum $\sigma(T)$ are isolated eigenvalues of finite multiplicities, in other words, the spectrum $\sigma(T)$ is at most a denumerable set having no accumulation points apart from zero. If $0 \neq \lambda \in \sigma(T)$, then the chain

$$
\{0\}=: \mathcal{N}_{\lambda}^{0}(T) \subset \mathcal{N}_{\lambda}^{1}(T) \subset \ldots \subset \mathcal{N}_{\lambda}^{n}(T) \subset \ldots,
$$

where (the root subspaces)

$$
\mathcal{N}_{\lambda}^{k}:=\operatorname{ker}(T-\lambda I)^{k}, \quad k=1,2, \ldots,
$$

is stabilised. The least number $\nu:=\nu_{\lambda}(T)$ satisfying $\mathcal{N}_{\lambda}^{\nu+1}=\mathcal{N}_{\lambda}^{\nu}$ is called the order of the root subspaces $\mathcal{N}_{\lambda}^{k}$. The following (skew) decomposition holds

$$
\mathcal{X}=\mathcal{N}_{\lambda}^{\nu}+\mathcal{W}
$$

where, as already was pointed, $\mathcal{N}_{\lambda}^{\nu}$ is a finite-dimensional subspace in $\mathcal{X}$, and $\mathcal{W}$ being an invariant subspace of $T$. Moreover, the restriction of $T-\lambda I$ to $\mathcal{W}$ is an invertible operator.

The theorem on the invertibility of analytic operator-valued functions, usually in the literature, occurs in various forms and contexts, mainly for the case of Hilbert spaces. The questions connected with it go back to the work of J.D.Tamarkin [Tam27], but we refer to I.Gohberg [Goh51], see also [GKn69], Theorem 5.1, who was the first treating the problem (still remaining within Hilbert spaces) in an abstract framework. This treatment is more appropriate to our purposes. For some related material see [RS78], [RS75], [RS72] and [DS88]. Nevertheless, in view of our purposes, we need a version of it for the general case of Banach spaces. Such a result is due to Stanly Steinberg [Ste69]. We formulate this result and provide a proof according to our goals and applications. 
Theorem 1.3.2. (Analytic Fredholm Theorem). Let $\mathcal{X}$ be a Banach space, let $\Omega$ be an open and connected domain in the complex plane, and suppose that $T(\cdot)$ is an analytic operator-valued function defined on $\Omega$ with $T(z)$ compact operators in $\mathcal{X}$ for $z \in \Omega$. Then either $I-T(z)$ is nowhere invertible in $\Omega$ or else the inverse $(I-T(z))^{-1}$ exists for all $z \in \Omega$ except at a countable number of isolated points.

Proof. Denote $\Omega_{0}$ the set of all points $z$ in $\Omega$ such that the operator $I-T(z)$ is not invertible. $\Omega_{0}$ is a closed set in $\Omega$ since the set of invertible operators in $\mathcal{B}(X)$ is open. Now, if $z_{0} \in \Omega_{0}$, due to the fact that the (non-zero) eigenvalues of compact operator are isolated, we have that $T\left(z_{0}\right)-\lambda I$ is an invertible operator for all $\lambda$ sufficiently close to 1 . Hence a circle $C$ enclosing at most the point 1 in $\sigma\left(T\left(z_{0}\right)\right)$ and such that $T\left(z_{0}\right)-\lambda I$ to be invertible for all $\lambda \in C$. $T\left(z_{0}\right)-\lambda I$ is continuous in $\lambda$, can be chosen. This means that $C$ as a compact set is transformed by $\lambda \rightarrow T\left(z_{0}\right)-\lambda I$ into a compact set in the set of invertible operators in $\mathcal{B}(\mathcal{X})$. From this fact and that $T(z)$ depends analytically in $z$, it follows that $T(z)-\lambda I$ are also invertible operators (in $\mathcal{B}(\mathcal{X})$ ) for $\lambda \in C$ and $z$ sufficiently close to $z_{0}$. For those $z$ we can define

$$
P(z)=-\frac{1}{2 \pi i} \int_{C}(T(z)-\lambda I)^{-1} d \lambda
$$

$P(z)$ is a projection (Riesz projection) and, as a map depending on $z$, is analytic on a neighbourhood $\mathcal{V}_{0}$ of $z_{0}$. Furthermore, $P\left(z_{0}\right)$ is the projection on the root subspace, denote it by $\mathcal{N}_{1}$, of $T\left(z_{0}\right)$ corresponding to the eigenvalue 1 . We let

$$
\mathcal{X}=\mathcal{N}_{1}+\mathcal{W}
$$

for the corresponding (skew) decomposition. Both subspaces $\mathcal{N}_{1}$ and $\mathcal{W}$ are invariant for $T\left(z_{0}\right)$, and note that $I-T\left(z_{0}\right)$ considered as an operator on $\mathcal{W}$ is invertible.

Further, following Wolf [Wol52], we introduce the following auxiliary operator

$$
U(z)=P\left(z_{0}\right) P(z)+\left(I-P\left(z_{0}\right)\right)(I-P(z))
$$

for $z$ in the chosen neighborhood $\mathcal{V}_{0}$. Since $U\left(z_{0}\right)=I$ and since $U(z)$ depends analytically in $z$, without any restriction, it follows that $U(z)$ is invertible at each $z \in \mathcal{V}_{0}$. We set

$$
A(z)=U(z)(I-T(z)) U(z)^{-1}, \quad z \in \mathcal{V}_{0},
$$

and observe that the operator $P\left(z_{0}\right)$ is commuting with $A(z)$. In fact, since

$$
U(z) P(z)=P\left(z_{0}\right) P(z)=P\left(z_{0}\right) U(z),
$$

we have

$$
\begin{gathered}
P\left(z_{0}\right) A(z)=P\left(z_{0}\right) U(z)(I-T(z)) U(z)^{-1}= \\
=U(z) P(z)(I-T(z)) U(z)^{-1}= \\
=U(z)(I-T(z)) P(z) U(z)^{-1}= \\
=U(z)(I-T(z)) U(z)^{-1} P\left(z_{0}\right)=A(z) P\left(z_{0}\right),
\end{gathered}
$$


i.e.,

$$
P\left(z_{0}\right) A(z)=A(z) P\left(z_{0}\right) .
$$

Therefore, the operator $A(z)$ is decomposed as a sum of two operators acting respectively on $\mathcal{N}_{1}$ and $\mathcal{W}$. Let $A(z) / \mathcal{N}_{1} A(z) / \mathcal{W}$ denote the restrictions of $A(z)$ to $\mathcal{N}_{1}$ and $\mathcal{W}$, respectively. Since $A(z)$ is close to $A\left(z_{0}\right)=I-P\left(z_{0}\right)$, and since $I-T\left(z_{0}\right)$ is invertible in $\mathcal{W}$, it follows that $A(z) / \mathcal{W}$ is also an invertible operator. The root subspace $\mathcal{N}_{1}$ is of finite dimension, so the restriction $A(z) / \mathcal{N}_{1}$ is a finite rank operator. There are two possibilities, either $\operatorname{det} A(z) / \mathcal{N}_{1}$ is identically equal to zero on $\mathcal{V}_{0}$ or else it becomes zero only at isolated points. In the first case $z_{0}$ belongs to $\Omega_{0}$ together with a neighborhood $\mathcal{V}_{0}$. This means that $\Omega_{0}$ is an open set and, since it is also closed in $\Omega$ and $\Omega$ is connected, it follows that either $\Omega_{0}=\varnothing$ or $\Omega_{0}=\Omega$. If the second case occurres, then the operator-valued function $I-T(z)$ is not invertible only on a denumerable set of isolated points.

The analytic Fredholm theorem is naturally involved in the factorization scheme presented in Section 1.2. In the assumptions 1.2.1, 1.2.2, 1.2.3 and 1.3.1 one can take $-Q(z)$ instead of $T(z)$. Then the conditions of Theorem 1.3 .2 are satisfied in the sense that in the resolvent set $\rho\left(H_{0}\right)$ of the unperturbed operator $H_{0}$ there can be at most a countable set of isolated points $z$ for which the operator $I+Q(z)$ is not invertible. By virtue of Theorem 1.2.10 these points are eigenvalues of the perturbed operator $H$ defined by (1.2). In our concrete applications to the class of Schrödinger type operators and Dirac operators, the unperturbed operator $H_{0}$ appears only with a component of the continuous spectrum, even absolutely continuous (as, for instance, in the case of Schrödinger operators $-\Delta+V(x)$ the unperturbed operator $H_{0}$ is $-\Delta$, the Laplacian, with its essential spectrum the interval $[0, \infty))$. These are the situations where the analytic Fredholm theorem can be applied directly to conclude that the essential spectra of $H_{0}$ and $H$ coincide. It is worth mentioning that, under our assumptions, the same fact on the coincidence of the essential spectra remains true also for the case where the unperturbed operator $H_{0}$ has a nontrivial part of the discrete spectrum. In such cases a more general theorem is required with the implication of meromorphic functions instead of the analytical ones. The arguments used in the proof of the analytic Fredholm theorem (Theorem 1.3.2) are applicable to the general case of meromorphic functions. Note that we treat the problem only with regard to operator-valued analytic functions because of the applications we are considering.

In general, the following result holds true.

Theorem 1.3.3. Under the assumptions 1.2.1, 1.2.2, 1.2.3 and 1.3.1 the essential spectra of $H_{0}$ and $H$ coincide.

The result given by Theorem 1.3.3 may be regarded as being a unification of Weyl's type theorems concerning the invariance of the essential spectrum under relatively compact perturbations. The Weyl's type theorems, and also other stability theorems used frequently in perturbation theory [Kat95], can be derived directly from the general results presented in the outlined abstract scheme framework. In particular, when $B$ is taken as $V$ and $A$ as $I$ we have in fact that 
$D(V) \supset D\left(H_{0}\right)$ and $V R\left(z ; H_{0}\right)$ is a compact operator on $\mathcal{X}$ for some (or, equivalently, all) $z \in \rho\left(H_{0}\right)$. This means that the operator $V$ is relatively compact with respect to $H_{0}$. Then, as is well-known (see, for instance, [Kat95] or also [Sch86]), both operators $H_{0}$ and $H$ are defined on the same domain being closed operators, simultaneously. Furthermore, they have the same essential spectrum (for the case of Banach spaces see [Sch86], Theorem 4.6). In the case of Hilbert spaces if $H_{0}$ is self-adjoint and $V$ a symmetric relatively compact with respect to $H_{0}$, then $H=H_{0}+V$, it is a self-adjoint operator and has the same essential spectrum as $H_{0}$. 


\section{2 - Discussion of Paper I}

\subsection{Overview of Paper I}

The paper is devoted to the problem of localization of eigenvalues for Schrödinger type operators. Evaluations for the eigenvalues are made in terms of norms of Lebesgue's classes. Slowly decaying potentials are also considered. The methods applied allow studying the problem for non-self-adjoint operators even considered acting in one of the Lebesgue spaces $L_{p}\left(\mathbb{R}^{n}\right)(1<p<\infty)$. The results cover and improve those known previously, in particular, those of Frank [Fra11], Laptev and Safronov [LS09], and Safronov [Saf10]. We mention also [Dav02] for an overview on certain aspects of spectral analysis of non-self-adjoint operators mainly needed for problems in quantum mechanics.

\subsubsection{Schrödinger Operators}

The Schrödinger operators are among the most important classes of Hamiltonians that arise in nonrelativistic quantum mechanics. These operators are of the form (apart from a numerical factor and appropriate units)

$$
H=-\Delta+q(x), \quad x \in \mathbb{R}^{n},
$$

where $\Delta$ is the $n$-dimensional Laplacian, and (the potential) $q$ is a complex-valued measurable function on $\mathbb{R}^{n}$. Even though, Hilbert spaces case is the most important from the application point of view, we allow ourselves to consider the Schrödinger operators to be defined on Lebesgue spaces $L_{p}\left(\mathbb{R}^{n}\right)(1<p<\infty)$. We denote by $H_{0}$ the operator $-\Delta$ considered defined in $L_{p}\left(\mathbb{R}^{n}\right)$ on its domain the Sobolev space $W_{p}^{2}\left(\mathbb{R}^{n}\right)$. The potential $q$ is the operator of multiplication $q: u \rightarrow q(x) u$, where $u \in L_{p}\left(\mathbb{R}^{n}\right)$ such that $q u \in L_{p}\left(\mathbb{R}^{n}\right)$. The spectral theory of such partial differential operators in Banach spaces is also of a great importance in applications, occurs in diverse contexts, and is treated quite intensively at this time. We refer only to [Sch86] and references cited therein, considering this treatment as sufficient for our purposes.

It is found that the unperturbed operator $H_{0}(=-\Delta)$, as defined above, is closed, and its spectrum $\sigma\left(H_{0}\right)$, independently of the value of $p$, is the interval $[0, \infty)$. As in the case of the Hilbert space $p=2$, the operator $H_{0}$ does not have eigenvalues. Conditions for the perturbation operator, i.e., potential, are assumed to give an appropriate definition for Schrödinger operators so that to be also closed and have the same essential spectrum as $H_{0}$. We assume that the potential $q$ admits a factorization $q=a b$ with $a \in L_{r}\left(\mathbb{R}^{n}\right)$ and $b \in L_{s}\left(\mathbb{R}^{n}\right)$ for some $0<r, s<\infty$. We let $A$ and $B$ denote the multiplication operators with $a$ and $b$, respectively, defined in $L_{p}\left(\mathbb{R}^{n}\right)$ on their maximal domains. To ensure $H_{0^{-}}$ boundedness of $A$ and $B$ we let $a$ and $b$ be functions of Stummel classes [Stu56] 
(see also [JW73] and [Sch86]), for instance, namely

$$
\begin{array}{cl}
M_{\nu, p^{\prime}}(a)<\infty, & 0<\nu<p^{\prime}, \\
M_{\mu, p}(b)<\infty, & 0<\mu<p,
\end{array}
$$

where $p^{-1}+p^{\prime-1}=1$ and

$$
M_{\nu, p}(u)=\sup _{x} \int_{|x-y|<1}|u(y)|^{p}|x-y|^{\nu-n} d y
$$

for functions $u \in L_{p, l o c}\left(\mathbb{R}^{n}\right)$. Thereby, the $H_{0}$-boundedness of $A$ and $B$ is ensured, cf. [Sch86], Theorem 2.1, Ch.6, page 105 or, what is the same, $D(A) \cap D(B) \supset$ $W_{p}^{2}\left(\mathbb{R}^{n}\right)$ or else $A$ and $B$ are bounded operators from $W_{p}^{2}\left(\mathbb{R}^{n}\right)$ to $L_{p}\left(\mathbb{R}^{n}\right)$. So, under these conditions, the bordered resolvent $B R\left(z ; H_{0}\right) A=B\left(H_{0}-z I\right)^{-1} A$ is a densely defined operator and has a (unique) bounded extension $Q(z)$ for $z \in \rho\left(H_{0}\right)$. If, in addition, $q$ is somewhat small at infinity, for instance it will be enough to let

$$
\int_{|x-y|<1}|b(y)|^{p} d y \rightarrow 0 \quad \text { as } \quad|x| \rightarrow \infty,
$$

then $Q(z)$ is a compact operator. In this way, the results given in the abstract framework of Section 1.2 and 1.3 can be applied, in accordance with the operator $H_{0}+A B(=-\Delta+q)$ admits a closed extension $H$ having the same essential spectrum as unperturbed operator $H_{0}(=-\Delta)$.

In what follows, we take the operator $H$ as the Schrödinger operator corresponding to $-\Delta+q$ in above sense and we give estimates for the eigenvalues of $H$ located outside the essential spectrum. The problem reduces to estimation of the resolvent of the unperturbed operator $H_{0}$, that is defined by $-\Delta$ in $L_{p}\left(\mathbb{R}^{n}\right)$ on its domain the Sobolev space $W_{p}^{2}\left(\mathbb{R}^{n}\right)$, bordered by some suitable operators of multiplication. This approach is based on the arguments pointed out in the framework of the factorization scheme presented in Section 1.2. So, we proceed as follows. We evaluate the resolvent operator $R\left(z ; H_{0}\right)$ and, depending on the result, choose an appropriate factorization of the potential $q=a b$ so that the bordered resolvent $B R\left(z ; H_{0}\right) A$ has a bounded extension $Q(z)$. In virtue of Theorem 1.2.10 it must be fulfilled $\|Q(\lambda)\| \geq 1$ for eigenvalues $\lambda$. From this inequality we get the desired estimates for eigenvalues.

We begin with evaluation of perturbed eigenvalues situated on the left halfplane $\operatorname{Re} z<0$. Therewith, in particular, bounds of the negative eigenvalues for the self-adjoint case, mostly which is important in applications, are established.

Theorem 2.1.1. Let $1<p<\infty$, and let $q=a b$, where $a \in L_{r}\left(\mathbb{R}^{n}\right)$ and $b \in$ $L_{s}\left(\mathbb{R}^{n}\right)$ with $0<r \leq \infty, p \leq s \leq \infty$ and $r^{-1}+s^{-1}<2 n^{-1}$. Then, for eigenvalues $\lambda$ with $\operatorname{Re} \lambda<0$ of the Schrödinger operator $H$, considered acting in the space $L_{p}\left(\mathbb{R}^{n}\right)$, it holds that

$$
|\operatorname{Re} \lambda|^{1-n / 2 \alpha^{\prime}} \leq C(n, r, s)\|a\|_{r}\|b\|_{s}
$$

where

$$
C(n, r, s)=(4 \pi)^{-n / 2 \alpha^{\prime}} \alpha^{-n / 2 \alpha} \Gamma\left(1-n / 2 \alpha^{\prime}\right), \quad \alpha=\left(1-r^{-1}-s^{-1}\right)^{-1} .
$$


In the proofs we use the representation of the free Green function $G_{n}(x, y ; \lambda)$. In three dimensional case $n=3$ it is the function

$$
G_{3}(x, y ; \lambda)=\frac{1}{4 \pi|x-y|} e^{i \sqrt{\lambda}|x-y|},
$$

where the square root $\sqrt{\lambda}$ of $\lambda$ is taken with positive imaginary part. So, the resolvent of $H_{0}(=-\Delta)$ is the following integral operator

$$
\left(R\left(\lambda ; H_{0}\right) u\right)(x)=\frac{1}{4 \pi} \int_{\mathbb{R}^{3}} \frac{e^{i \sqrt{\lambda}|x-y|}}{|x-y|} u(y) d y, \quad u \in L_{p}\left(\mathbb{R}^{3}\right) .
$$

For the general case of an arbitrary dimension, the fundamental solution of the Laplacian, and therefore the kernel of the resolvent $R\left(\lambda ; H_{0}\right)$, is expressed by Bessel's functions (see, for instance, [BS91]). The direct use of such a representation encounters considerable difficulties on the calculations, due to of which, we make use the following integral representation (the heat kernel) of the free Green function

$$
G_{n}(x, y ; \lambda)=(4 \pi)^{-n / 2} \int_{0}^{\infty} e^{\lambda t} e^{-|x-y|^{2} / 4 t} t^{-n / 2} d t, \quad \operatorname{Re} \lambda<0 .
$$

In this respect, we refer to [Dav89].

The eigenvalues of $H$ in the right half-plane, where the essential spectrum is contained, are also of particular interest. These are scattered around the essential spectrum and, thus, the question is to establish, as optimally as possible, the band in which they are located. So, we have to evaluate their imaginary parts. For this purpose, the same arguments are applied, but in this case, it is more convenient to use the formula expressing the resolvent operator $R\left(\lambda ; H_{0}\right)$ by means of the Laplace transform of the operator-group $e^{-i t H_{0}}(-\infty<t<\infty)$

$$
\begin{aligned}
R\left(\lambda ; H_{0}\right)=i \int_{0}^{\infty} e^{i \lambda t} e^{-i t H_{0}} d t, & \operatorname{Im} \lambda>0, \\
=-i \int_{-\infty}^{0} e^{i \lambda t} e^{-i t H_{0}} d t, & \operatorname{Im} \lambda<0
\end{aligned}
$$

(see, for instance, [HP74]). As is known (see [Kat95], Ch. IX, pag. 496, or, also [Pro64]) for a fixed real $t, e^{-i t H_{0}}$ represents an integral operator (in a generalized sense) with the kernel

$$
h(x, y ; t)=(4 \pi i t)^{-n / 2} e^{-|x-y|^{2} / 4 i t} .
$$

In quantum physics the kernel function $h(x, y ; t)$ is known as the free propagator.

We obtain the following result.

Theorem 2.1.2. Let $1<p<\infty$, and let $q=a b, \quad a \in L_{r}\left(\mathbb{R}^{n}\right), \quad b \in L_{s}\left(\mathbb{R}^{n}\right)$ for $r, s$ satisfying $0<r \leq \infty, p \leq s \leq \infty, r^{-1}-s^{-1}=1-2 p^{-1}, 2^{-1}-p^{-1} \leq r^{-1} \leq$ $1-p^{-1}$, 
$r^{-1}+s^{-1}<2 n^{-1}$. Then, for any complex eigenvalue $\lambda$ with $\operatorname{Im} \lambda \neq 0$ of the Schrödinger operator $H$, considered acting in the space $L_{p}\left(\mathbb{R}^{n}\right)$, there holds

$$
|\operatorname{Im} \lambda|^{\alpha} \leq(4 \pi)^{\alpha-1} \Gamma(\alpha)\|a\|_{r}\|b\|_{s}
$$

where $\alpha=1-n\left(r^{-1}+s^{-1}\right) / 2$.

The evaluation of eigenvalues can be carried out in the same framework of the factorization scheme by application of the Fourier transform map $F$. Recall that the Fourier transform map is defined by

$$
(F u)(\xi)=(2 \pi)^{-n / 2} \int_{\mathbb{R}^{n}} u(x) e^{-i x \cdot \xi} d x
$$

$\left(x \cdot \xi=x_{1} \xi_{1}+\ldots+x_{n} \xi_{n}\right.$ denotes the scalar product of $x, \xi$ in $\left.\mathbb{R}^{n}\right)$ at first on functions $u$ from the Schwartz class $\mathcal{S}\left(\mathbb{R}^{n}\right)$ and then extended to the space of tempered distributions $\mathcal{S}^{\prime}\left(\mathbb{R}^{n}\right)$ (the dual of $\mathcal{S}\left(\mathbb{R}^{n}\right)$ ). For the details we cite [RS75], Ch. IX, and, also, [Gru09], Ch. 5. Note that functions $u$ in $L_{p}\left(\mathbb{R}^{n}\right)(1 \leq p \leq \infty)$ are contained in $\mathcal{S}^{\prime}\left(\mathbb{R}^{n}\right)$, and so $\hat{u}:=F u$ are well-defined elements of $\mathcal{S}^{\prime}\left(\mathbb{R}^{n}\right)$.

For any $\lambda \in \mathbb{C} \backslash[0, \infty)$ we write

$$
\left.\widehat{R\left(\lambda ; H_{0}\right.}\right)=F R\left(\lambda ; H_{0}\right) F^{-1},
$$

and observe that $\left.R \widehat{R\left(\lambda ; H_{0}\right.}\right)$ represents the multiplication operator by $\left(|\xi|^{2}-\lambda\right)^{-1}$, i.e.,

$$
\left.\left(\widehat{R\left(\lambda ; H_{0}\right.}\right) \hat{u}\right)(\xi)=\left(|\xi|^{2}-\lambda\right)^{-1} \hat{u}(\xi), \quad \xi \in \mathbb{R}^{n} .
$$

On $L_{2}\left(\mathbb{R}^{n}\right)$ the mentioned relation is true. We use it for the space $L_{p}\left(\mathbb{R}^{n}\right)$ with

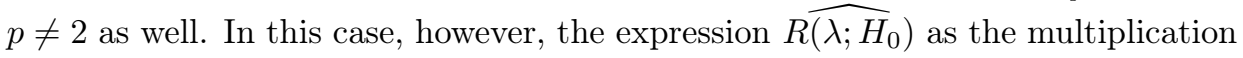
operator should be considered by definition.

We have the following result.

Theorem 2.1.3. Let $1<p<\infty$, and let $q=a b$, where $a \in L_{r}\left(\mathbb{R}^{n}\right)$ and $b \in$ $L_{s}\left(\mathbb{R}^{n}\right)$ for $r, s$ satisfying $0<r \leq \infty, 0<s \leq \infty, 2^{-1}-p^{-1} \leq r^{-1} \leq 1-p^{-1}$, $p^{-1}-2^{-1} \leq s^{-1} \leq p^{-1}$, and $r^{-1}+s^{-1}<2 n^{-1}$. Then, for any eigenvalue $\lambda \in \mathbb{C} \backslash[0, \infty)$ of the Schrödinger operator $H$, considered acting in the space $L_{p}\left(\mathbb{R}^{n}\right)$, the estimation

$$
|\lambda|^{\alpha-n / 2} \leq C(n, \alpha, \theta)\|a\|_{r}^{\alpha}\|b\|_{s}^{\alpha}
$$

holds, with

$C(n, \alpha, \theta)=(4 \pi)^{-n / 2}(2 \sin \theta)^{\alpha / 2-1 / 2} \Gamma(\alpha / 2+1) \Gamma(\alpha-n / 2) \Gamma(\alpha)^{-1} P_{n / 2-\alpha / 2-1 / 2}^{1 / 2-\alpha / 2}(-\cos \theta)$,

where $\alpha=\left(r^{-1}+s^{-1}\right)^{-1}$, and $P_{\mu}^{\nu}(z)(-1 \leq z \leq 1)$ denote the Gegenbauer polynomials.

For the Gegenbauer polynomials see [GR07]; 8.7-8.8.

From the results given by Theorems 2.1.1, 2.1.2 and 2.1.3 many particular estimates useful in applications can be derived. For instance, if in Theorem 2.1.1 
we let $r=s=2 \gamma+n$ with $\gamma>0$ for $1<p \leq n$ and $2 \gamma \geq p-n$ for $p>n$, and $a(x)=|q(x)|^{1 / 2}, b(x)=(\operatorname{sgn} q(x))|q(x)|^{1 / 2}$, where $\operatorname{sgn} q(x)=q(x) /|q(x)|$ if $q(x) \neq 0$ and $\operatorname{sgn} q(x)=0$ if $q(x)=0$, then we get

$$
|\operatorname{Re} \lambda|^{\gamma} \leq C(n, \gamma) \int_{\mathbb{R}^{n}}|q(x)|^{\gamma+n / 2} d x, \quad \operatorname{Re} \lambda<0,
$$

with a constant $C(n, \gamma)$ as in Theorem 2.1.1 expressed correspondingly by $n$ and $\gamma$.

Note that Frank [Fra11] also obtains a result similar to that already mentioned, but for the case of the Hilbert space $L_{2}\left(\mathbb{R}^{3}\right)$ under the restriction $0<\gamma<3 / 2$. The proofs in [Fra11] are based on a uniform Sobolev inequality due to Kenig et al. [KRS87].

Another type of estimates can be obtained directly from the results mentioned above by involving decaying potentials. Taking

$$
a(x)=\left(1+|x|^{2}\right)^{-\tau / 2} \quad \text { and } \quad b(x)=\left(1+|x|^{2}\right)^{\tau / 2} q(x)
$$

with $\tau r>n$, then $a \in L_{r}\left(\mathbb{R}^{n}\right)$ and,

$$
\|a\|_{r}^{r}=\pi^{n / 2} \Gamma((\tau r-n) / 2) / \Gamma((\tau r) / 2) .
$$

Now, supposing that

$$
\left(1+|x|^{2}\right)^{\tau / 2} q \in L_{r}\left(\mathbb{R}^{n}\right),
$$

we derive, for instance, from the result given by Theorem 2.1.3, the following estimate

$$
|\lambda|^{r-n} \leq C \int_{\mathbb{R}^{n}}\left|\left(1+|x|^{2}\right)^{\tau / 2} q(x)\right|^{r} d x
$$

with an appropriate constant $C$, and under relevant restrictions on $\tau$ and $r$.

Estimates of type (2.2) can be given by choosing other weight functions, used frequently for diverse purposes, as, for instance, $e^{\tau|x|}, e^{\tau|x|^{2}},|x|^{\alpha} e^{\tau|x|^{\beta}}$, etc.

We refer to [Fra11] and [Saf10] for some related results concerning eigenvalue estimates for Schrödinger operators with slowly decaying potentials. In this respect see also [DN02].

\subsubsection{Polyharmonic Operators}

The results discussed in the previous subsection admit extension to a more general case of polyharmonic operators. These are operators of the form

$$
H=(-\Delta)^{m}+q
$$

where (the potential) $q$ is a complex-valued function, and $m$ is an arbitrary positive number. The unperturbed operator $H_{0}=(-\Delta)^{m}$ can be treated upon unitary equivalence (by the Fourier transform) as the operator of multiplication by $|\xi|^{2 m}$. In the space $L_{p}\left(\mathbb{R}^{n}\right)$ we consider $H_{0}$ defined on domain the Sobolev space $W_{p}^{2 m}\left(\mathbb{R}^{n}\right)$. As in the usual case of Schrödinger operators, we assume that the potential $q$ admits a factorization $q=a b$ with $a$ and $b$ being function of Stummel 
classes, i.e., $M_{\nu, p^{\prime}}(a)<\infty$ and $M_{\mu, p}(b)<\infty$, but now with $0<\nu<p^{\prime} k$ and $0<\mu<p(m-k)$ for some $0<k<m$. Conditions on the potential $q$, in an average sense, to be small at infinity are also assumed. Under these conditions the operator $(-\Delta)^{m}+q$ admits a closed extension, we denote it by $H_{m, q}$, having the same essential spectrum as $H_{0}$. We have again $\sigma_{\text {ess }}\left(H_{0}\right)=[0, \infty)$, independently of values $p$. The problem of evaluation of perturbed eigenvalues (lying outside the essential spectrum) reduces in the abstract framework of the factorization scheme described in Section 1.2 and 1.3 to resolvent estimation of the unperturbed operator bordered by the corresponding operators of multiplication $A$ and $B$ (defined as in the previous subsection).

The main result is the following.

Theorem 2.1.4. Let $1<p<\infty, m>0$, and let $q=a b$, where $a \in L_{r}\left(\mathbb{R}^{n}\right)$, $b \in L_{s}\left(\mathbb{R}^{n}\right)$ for $r, s$ satisfying $0<r \leq \infty, 0<s \leq \infty, 2^{-1}-p^{-1} \leq r^{-1} \leq 1-p^{-1}$, $p^{-1}-2^{-1} \leq s^{-1} \leq p^{-1}$, and $r^{-1}+s^{-1}<2 m / n$. Then, for any eigenvalue $\lambda=|\lambda| e^{i \theta}(0<\theta<2 \pi)$ of the operator $H_{m, q}$, considered acting in $L_{p}\left(\mathbb{R}^{n}\right)$, there holds

$$
|\lambda|^{\alpha-n / 2 m} \leq C(n, m, \alpha, \theta)\|a\|_{r}^{\alpha}\|b\|_{s}^{\alpha},
$$

where $\alpha=\left(r^{-1}+s^{-1}\right)^{-1}$.

The constant in (2.3) is given by

$$
C(n, m, \alpha, \theta)=(4 \pi)^{-n / 2}(m \Gamma(n / 2))^{-1} I_{m}(n, \alpha, \theta),
$$

where

$I_{m}(n, \alpha, \theta)=(2 \sin \theta)^{\alpha / 2-1 / 2} \Gamma(\alpha / 2+1 / 2) B(n / 2 m, \alpha-n / 2 m) P_{n / 2 m-\alpha / 2-1 / 2}^{1 / 2-\alpha / 2}(-\cos \theta)$.

Here, $B(x, y)$ denotes the beta function.

An immediate consequence of Theorem 2.1.4 is the following, an estimate analogous to that given by $(2.1)$ :

Corollary 2.1.5. Let $\gamma>0$ for $n>2 m$ and $\gamma \geq 1-n / 2 m$ for $n<2 m$. If

$$
q \in L_{\gamma+n / 2 m}\left(\mathbb{R}^{n}\right),
$$

then every eigenvalue $\lambda=|\lambda| e^{i \theta}(0<\theta<2 \pi)$ of the operator $H_{m, q}$ satisfies

$$
|\lambda|^{\gamma} \leq C(n, m, \alpha, \theta) \int_{\mathbb{R}^{n}}|q(x)|^{\gamma+n / 2 m} d x
$$

where $C(n, m, \alpha, \theta)$ is as in $(2.3)$.

\subsubsection{Weak Lebesgue potentials}

The results discussed previously were obtained on the basis of resolvent estimates of the unperturbed operator $H_{0}$ (in both cases of Schrödinger and polyharmonic operators). In turn, the estimates for the resolvent operator are obtained essentially by applying the Riesz-Thorin interpolation theorem. This approach is quite 
suitable for the case when the potential is considered of the Lebesgue classes. However, it is also interesting to look at the problem for more general cases of potentials having singularities of another order such as those from weak Lebesgue classes. In such situations the Riesz-Thorin theorem generally becomes insufficient and cannot be applied. In our approach, the desired interpolation can be achieved, but using a more refined interpolation technique as, in particular, general Marcinkiewicz type theorems [BL76] (see also [Tri78]). In this more general setting, the so-called Lorentz spaces, representing generalizations of the usual Lebesgue spaces, are involved.

The weak $L_{r, w}\left(\mathbb{R}^{n}\right)$ spaces consists of all measurable, almost everywhere finite, complex-valued functions on $\mathbb{R}^{n}$ such that

$$
\|f\|_{r, \infty}:=\sup _{t>0}\left(t^{r} \lambda_{f}(t)\right)^{1 / r}<\infty
$$

where $\lambda_{f}$ denotes the distribution function of $|f|$, namely,

$$
\lambda_{f}(t)=\mu\left(\left\{x \in \mathbb{R}^{n}:|f(x)|>t\right\}\right), \quad 0<t<\infty,
$$

( $\mu$ is the standard Lebesgue measure on $\mathbb{R}^{n}$ ). The weak $L_{r}$-spaces are particular cases of the more general Lorentz classes $L_{p, r}\left(\mathbb{R}^{n}\right)$ (see, e.g., [BL76]). For a (fixed) pair $(p, r)(0<p<\infty, 0<r \leq \infty) L_{p, r}\left(\mathbb{R}^{n}\right)$ is defined as the space of all measurable functions $f$ on $\mathbb{R}^{n}$ for which

$$
\|f\|_{p, r}^{r}:=\int_{\mathbb{R}^{n}} t^{r}\left(\lambda_{f}(t)\right)^{r / p} \frac{d t}{t}<\infty .
$$

Note that $L_{r, r}\left(\mathbb{R}^{n}\right)=L_{r}\left(\mathbb{R}^{n}\right)$, and it will be convenient to let $L_{\infty, r}\left(\mathbb{R}^{n}\right)=$ $L_{\infty}\left(\mathbb{R}^{n}\right)(0<r \leq \infty)$.

Next, we consider the Schrödinger operator $H$ generated by $-\Delta+q(x)$ with a potential $q$ written as a product $q=a b$, where $a \in L_{r, w}\left(\mathbb{R}^{n}\right)$ and $b \in L_{s, w}\left(\mathbb{R}^{n}\right)$. Denote by $A, B$ the operators of multiplication by $a, b$, respectively, and proceed as follows. First, applying interpolation methods [BL76] in combination with a result due to O'Neil [O'N63], we show that the resolvent operator $R\left(\lambda ; H_{0}\right)$ is acting boundedly from $L_{\beta, p}\left(\mathbb{R}^{n}\right)$ to $L_{\gamma, p}\left(\mathbb{R}^{n}\right)$ for some suitable $\beta$ and $\gamma$. Then, in view of $a \in L_{r, w}\left(\mathbb{R}^{n}\right)$ and $b \in L_{s, w}\left(\mathbb{R}^{n}\right)$, the same O'Neil's result can be applied again and, under certain restrictions on $r$ and $s$, we get that $A$ is bounded as an operator regarded acting from $L_{p, p}\left(\mathbb{R}^{n}\right)$ to $L_{\beta, p}\left(\mathbb{R}^{n}\right)$ and, respectively, $B$ from $L_{\gamma, p}\left(\mathbb{R}^{n}\right)$ to $L_{p, p}\left(\mathbb{R}^{n}\right)$. Following the evaluations effectuated, we finally get a bound for the bordered resolvent, namely,

$$
\left\|B R\left(\lambda ; H_{0}\right) A\right\| \leq C I(n, \alpha, \theta)|\lambda|^{n / 2 \alpha-1}\|a\|_{r, \infty}\|b\|_{s, \infty}
$$

where $I(n, \alpha, \theta)(\theta:=\arg \lambda, 0<\theta<2 \pi)$ is as in (2.3) and $C$ is a positive constant depending only on $r, s$ and $p$ occurred after interpolation.

In this way, under restriction on $p, r$ and $s$ as in Theorem 2.1.4, any eigenvalue $\lambda \in \mathbb{C} \backslash[0, \infty)$ of the Schrödinger operator $H$ satisfies

$$
|\lambda|^{1-n / 2 \alpha} \leq C I(n, \alpha, \theta)\|a\|_{r, \infty}\|b\|_{s, \infty} .
$$


This result extends to the case of the polyharmonic operator $H_{m, q}$.

From the general results obtained one can also derive, as in the usual case of potentials from Lebesgue classes, various eigenvalue estimates useful in applications. 


\section{3 - Discussion of Paper II}

\subsection{Overview of Paper II}

In this paper we consider the problem of location of the discrete spectrum for Schrödinger operators on the half-line (in general) with complex-valued potentials. Bounds for the eigenvalues, lying outside the essential spectrum, are established in terms of $L_{p}$-norms of potentials. Schrödinger operators with potentials belonging to weak Lebesgue classes are also considered. The results cover and improve those known previously due to R. L. Frank, A. Laptev and R. Seiringer [FLS11].

The history of the problem is as follows. In Keller's work [Kel61] it is pointed an inequality for negative eigenvalues of the 1D (self-adjoint) Schrödinger operator $H$ $\left(=-d^{2} / d x^{2}+q(x)\right)$ considered on the space $L_{2}\left(\mathbb{R}^{n}\right)$. Assuming that the potential $q$ is a real-valued summable function on $\mathbb{R}$, it is shown in [Kel61] that any negative eigenvalue $\lambda$ of $H$ satisfies

$$
|\lambda|^{1 / 2} \leq \frac{1}{2} \int_{-\infty}^{\infty}|q(x)| d x
$$

It is known that the constant $1 / 2$ in $(3.1)$ is optimal. This result is extended in [AAD01] to the general case of non-self-adjoint Schrödinger operators with potentials belonging to $L_{1}\left(\mathbb{R}^{n}\right) \cap L_{2}\left(\mathbb{R}^{n}\right)$. In this context, it is worth mentioning that recently special attention has been paid to the study of non-self-adjoint Schrödinger operators because of the requirements of the quantum theory and analysis itself. We refer to [Dav02] for a comprehensive overview on certain aspects of spectral analysis of non-self-adjoint operators mainly needed with this respect.

In the recent paper [FLS11] this problem is considered for the case of $1 \mathrm{D}$ Schrödinger operators on the half-line with complex-valued potentials. As the main result in [FLS11] it is shown that any eigenvalue $\lambda=|\lambda| e^{i \theta} \in \mathbb{C} \backslash[0, \infty)$ $(0<\theta<2 \pi)$ of the Schrödinger operator $H$ in $L_{2}\left(\mathbb{R}_{+}\right)\left(\mathbb{R}_{+}:=[0, \infty)\right)$ with Dirichlet boundary conditions satisfies

$$
|\lambda|^{1 / 2} \leq \frac{1}{2} g(\cot (\theta / 2)) \int_{0}^{\infty}|q(x)| d x,
$$

where

$$
g(t):=\sup _{y>0}\left|e^{i t y}-e^{-y}\right| .
$$

This result is extended to the general case of the Schrödinger operators with mixed boundary conditions $u^{\prime}(0)=\sigma u(0) \quad(\sigma \geq 0)$. Instead of $(3.2)$, in this case, it is valid the following one

$$
|\lambda|^{1 / 2} \leq \int_{0}^{\infty}|q(x)| d x
$$


As is pointed out in [FLS11] the bound in (3.4) is optimal for any $0 \leq \sigma<\infty$ and any fixed argument $\theta, 0<\theta<2 \pi$, of the eigenvalue $\lambda$.

We study the problem within the framework of the abstract scheme described in Sections 1.2 and 1.3. For we assume that the potential $q$ admits a factorization $q=a b$, where $a \in L_{r}\left(\mathbb{R}_{+}\right)$and $b \in L_{s}\left(\mathbb{R}_{+}\right)$for some $0<r, s<\infty$. As in the multidimensional case (Section 2) additional subordinate conditions on the potential $q$ (in fact on $a$ and $b$ ) are required in order to guarantee the existence of a closed extension $H$ of the operator $-d^{2} / d x^{2}+q$, a priori given on smooth functions, having the same essential spectrum as the unperturbed operator $H_{0}\left(=d^{2} / d x^{2}\right)$. First we analyze the case of the Dirichlet boundary condition. In this case, the unperturbed operator $H_{0}$ is considered in $L_{p}\left(\mathbb{R}_{+}\right)(1<p<\infty)$ with domain the Sobolev space $\stackrel{\circ}{W_{p}^{2}}\left(\mathbb{R}_{+}\right)$consisting of all functions $u \in L_{p}\left(\mathbb{R}_{+}\right)$such that $u, u^{\prime}$ are absolutely continuous on each finite interval of $\mathbb{R}_{+}$with $u^{\prime \prime} \in L_{p}\left(\mathbb{R}_{+}\right)$ and $u(0)=0$. The operator $H_{0}$ is closed and $\sigma\left(H_{0}\right)=[0, \infty)$. The resolvent $R\left(\lambda ; H_{0}\right)=\left(H_{0}-\lambda I\right)^{-1}, \lambda \in \rho\left(H_{0}\right)(=\mathbb{C} \backslash[0, \infty))$, is an integral operator with the kernel

$$
r(x, y ; \lambda)=-\frac{1}{2 i \mu}\left(e^{i \mu|x-y|}-e^{i \mu(x-y)}\right), \quad 0<x, y<\infty
$$

where $\mu=\lambda^{1 / 2}$ is taken with $\operatorname{Im} \mu>0$. The kernel $r(x, y ; \lambda)$ is a smooth enough function that decreases rapidly at infinity, due to which subtle estimates for the resolvent operator $R\left(\lambda ; H_{0}\right)$ can be established. To this end, we take $\alpha$, with $1 \leq \alpha<\infty$, and observe that

$$
\sup _{x>0}\|r(x, \cdot \lambda)\|_{\alpha} \leq 1 /|\mu|(\alpha \operatorname{Im} \mu)^{1 / \alpha},
$$

from which we derive by straightforward verification that the resolvent operator $R\left(\lambda ; H_{0}\right)$ is bounded regarded as an operator from $L_{\alpha^{\prime}}\left(\mathbb{R}_{+}\right)$to $L_{\infty}\left(\mathbb{R}_{+}\right)$, where $\alpha^{\prime}$ is the conjugate exponent to $\alpha$, i.e., $\alpha^{\prime}=\alpha(\alpha-1)^{-1}$, with the same bound $1 /|\mu|(\alpha \operatorname{Im} \mu)^{1 / \alpha}$ for norms in both cases. By applying the Riesz-Thorin interpolation theorem we conclude that the resolvent operator $R\left(\lambda ; H_{0}\right)$ is bounded from $L_{\beta}\left(\mathbb{R}_{+}\right)$to $L_{\gamma}\left(\mathbb{R}_{+}\right)$, with norm at most $1 /|\mu|(\alpha \operatorname{Im} \mu)^{1 / \alpha}$, for suitable $\beta$ and $\gamma$. Based on this estimation we can now easily evaluate the norm of the bordered resolvent $B R\left(\lambda ; H_{0}\right) A$ with $A$ and $B$ being the operators of multiplications by $a$ and $b$, respectively.

In this way we prove the following result.

Theorem 3.1.1. Let $1<p<\infty, 0<r \leq \infty, p \leq s \leq \infty, r^{-1}+s^{-1}<1$, and assume $q=a b$, where $a \in L_{r}\left(\mathbb{R}_{+}\right)$and $b \in L_{s}\left(\mathbb{R}_{+}\right)$. Then, for any eigenvalue $\lambda=|\lambda| e^{i \theta} \in \mathbb{C} \backslash \mathbb{R}_{+}(0<\theta<2 \pi)$ of the Schrödinger operator $H$, there holds

$$
|\lambda|^{1+\alpha} \leq(\alpha \sin (\theta / 2))^{-2}\|a\|_{r}^{2 \alpha}\|b\|_{s}^{2 \alpha}
$$

where $\alpha=\left(1-r^{-1}-s^{-1}\right)^{-1}$.

The following particular case is of special interest. 
Corollary 3.1.2. Let $\gamma>1 / 2$ if $1<p \leq 2$ and $2 \gamma \geq p-1$ if $p>2$, and suppose

$$
q \in L_{\gamma+1 / 2}\left(\mathbb{R}_{+}\right)
$$

Then any eigenvalue $\lambda=|\lambda| e^{i \theta} \in \mathbb{C} \backslash \mathbb{R}_{+}$of the operator $H$ in $L_{p}\left(\mathbb{R}_{+}\right)$satisfies

$$
|\lambda|^{\gamma} \leq\left(\frac{2 \gamma+1}{2 \gamma-1} \sin \frac{\theta}{2}\right)^{1 / 2-\gamma} \int_{0}^{\infty}|q(x)|^{\gamma+1 / 2} d x .
$$

In the self-adjoint case there can be only negative eigenvalues of the Schrödinger operator $H$, and thus (3.6) becomes

$$
|\lambda|^{\gamma} \leq\left(\frac{2 \gamma-1}{2 \gamma+1}\right)^{\gamma-1 / 2} \int_{0}^{\infty}|q(x)|^{\gamma+1 / 2} d x .
$$

Similar estimates for whole-line operators were pointed out in [Kel61] and [LT76]. For related results and discussion in other contexts see also [DN02] and [LS09].

For the limiting case when $\alpha=\infty$ we have

$$
\sup _{x>0}\|r(x, \cdot ; \lambda)\|_{\infty}=\sup _{x, y>0} \frac{1}{2|\mu|}\left|e^{i \mu|x-y|}-e^{i \mu(x+y)}\right| .
$$

This supremum has been computed in [FLS11] (see [FLS11], Lemma 1.3). It turns out that

$$
\sup _{x, y>0}\left|e^{i \mu|x-y|}-e^{i \mu(x+y)}\right|=g(\cot (\theta / 2)),
$$

where $g(t)$ is as in (3.3). Thus

$$
\sup _{x>0}\|r(x, \cdot ; \lambda)\|_{\infty}=\frac{1}{2|\mu|} g(\cot (\theta / 2)),
$$

and, therefore, the resolvent operator $R\left(\lambda ; H_{0}\right)$ is bounded from $L_{1}\left(\mathbb{R}_{+}\right)$to $L_{\infty}\left(\mathbb{R}_{+}\right)$, and

$$
\left\|R\left(\lambda ; H_{0}\right) v\right\|_{\infty} \leq \frac{1}{2|\mu|} g(\cot (\theta / 2)) \quad\|v\|_{1}, \quad v \in L_{1}\left(\mathbb{R}_{+}\right) .
$$

In this case, take $\beta=1, \gamma=\infty$, and $r=p^{\prime}, s=p$, and we have the following result:

Theorem 3.1.3. Let $1<p<\infty$, and let $q=a b$, with $a \in L_{p}\left(\mathbb{R}_{+}\right)$and $b \in$ $L_{p^{\prime}}\left(\mathbb{R}_{+}\right)$. Then any eigenvalue $\lambda=|\lambda| e^{i \theta} \in \mathbb{C} \backslash \mathbb{R}_{+}$of the Schrödinger operator $H$ in $L_{p}\left(\mathbb{R}_{+}\right)$satisfies

$$
|\lambda|^{1 / 2} \leq \frac{1}{2} g(\cot (\theta / 2))\|a\|_{p}\|b\|_{p^{\prime}} .
$$

In particular, for the Hilbert space case $p=2$, and $a(x)=|q(x)|^{1 / 2}, b(x)=$ $(\operatorname{sgn} q(x))|q(x)|^{1 / 2}$ the inequality (3.7) is nothing but (3.2) established in [FLS11] (it is presented by Theorem 1.1 [FLS11] as the main result).

By applying the same arguments there there can be obtained similar results concerning Schrödinger operators on the half-line subjected to (general) mixed 
boundary conditions $u^{\prime}(0)=\sigma u(0)(0 \leq \sigma<\infty$; in case $\sigma=\infty$ it is taken the Dirichlet conditions; $\sigma=0$ corresponds to the Neumann boundary conditions $\left.u^{\prime}(0)=0\right)$. The resolvent of the unperturbed operator, for this general case, is an integral operator with the kernel

$$
r_{\sigma}(x, y ; \lambda)=-\frac{1}{2 \mu i}\left(e^{i \mu|x-y|}-\frac{\sigma+i \mu}{\sigma-i \mu} e^{i \mu(x+y)}\right),
$$

where, as above, $\mu=\lambda^{1 / 2}$ is chosen so that $\operatorname{Im} \mu>0$.

It should be pointed out that the result given by Theorem 3.1.1 remain also valid, without any changes, for the general case of mixed boundary conditions. However, in the limiting case $\alpha=\infty$, we have

$$
\sup _{x>0}\left\|r_{\sigma}(x, \cdot ; \lambda)\right\|_{\infty}=\frac{1}{2|\mu|} g_{\sigma}(\cot (\theta / 2)),
$$

$g_{\sigma}$ instead of $g$, where

$$
g_{\sigma}(t):=\sup _{y>0}\left|e^{-i t y}-\frac{\sigma+i \mu}{\sigma-i \mu} e^{-y}\right|, \quad t \in \mathbb{R} .
$$

Accordingly, instead of (3.7) we have

$$
|\lambda|^{1 / 2} \leq \frac{1}{2} g_{\sigma}(\cot (\theta / 2))\|a\|_{p}\|b\|_{p^{\prime}} .
$$

Note that $g_{\sigma}(t)$ depends also on $\mu$ in a rather intricate way. However, it is uniformly bounded with respect to both variables $t$ and $\mu$, and moreover,

$$
g_{\sigma}(t):=\sup _{y>0}\left(1+e^{-y}\right)=2 .
$$

Thus, by (3.8), it follows

$$
|\lambda|^{1 / 2} \leq\|a\|_{p}\|b\|_{p^{\prime}}
$$

an inequality which for the Hilbert space case, that is, for $p=p^{\prime}=2$ and $a(x)=$ $|q(x)|^{1 / 2}, b(x)=\left(q(x)|\operatorname{sgn} q(x)|^{1 / 2}\right)$ was mentioned in [FLS11] (see Proposition 1.5 [FLS11]). In [FLS11] it is pointed that the inequality is sharp for any $\sigma \geq 0$ and any fixed argument $\theta, 0<\theta<2 \pi$ of the eigenvalue $\lambda$.

The results discussed above, in particular, the estimations (3.5), (3.6), (3.7) and (3.8), admit extensions to Schrödinger operators with potentials from the weak Lebesgue classes. 


\section{4 - Discussion of Paper III}

\subsection{Overview of Paper III}

Dirac operators, along with Schrödinger operators, have a decessive contribution to various areas of modern physics and mathematics. Spectral analysis of Dirac operators is of particular importance for solving many practical and theoretical problems. In view of the quantum-mechanical interpretation, the Dirac operators are usually considered in Hilbert spaces whose elements are related to the corresponding physical states. In this way, the operators are regarded as a result of certain identification with physical observables and treated like Hamiltonians corresponding to particles energies.

The tree-dimensional Dirac operator, frequently occurring as the main object in mathematical models of relativistic quantum mechanics, is given formally by the matrix-valued differential expression ([Foc78], [Tha92]).

$$
\mathfrak{h}(\psi)=i^{-1} \hbar c \alpha \cdot \nabla \psi+\beta m c^{2} \psi+V \psi,
$$

where

$$
\alpha \cdot \nabla=\sum_{j=1}^{3} \alpha_{j} \partial_{x_{j}}, \quad x=\left(x_{1}, x_{2}, x_{3}\right) \in \mathbb{R}^{3},
$$

$\partial_{x_{j}}:=\partial / \partial_{x_{j}}(j=1,2,3)$ are differentiation operators and, in standard representation,

$$
\beta=\left(\begin{array}{cc}
I_{2} & 0 \\
0 & -I_{2}
\end{array}\right), \quad \alpha_{j}=\left(\begin{array}{cc}
0 & \sigma_{j} \\
\sigma_{j} & 0
\end{array}\right), \quad j=1,2,3,
$$

$\sigma_{j}$ being the Pauli matrices

$$
\sigma_{1}=\left(\begin{array}{ll}
0 & 1 \\
1 & 0
\end{array}\right), \quad \sigma_{2}=\left(\begin{array}{cc}
0 & -i \\
i & 0
\end{array}\right), \quad \sigma_{3}=\left(\begin{array}{cc}
1 & 0 \\
0 & -1
\end{array}\right),
$$

$I_{2}$ denotes the identity $2 \times 2$ matrix; $m$ and $c$ denote the mass (of the particle) and the speed of light, respectively; $\hbar$ is the (reduced) Planck's constant (we take $\hat{h}=1$, in appropriate units). In (4.1) $\mathfrak{h}$ is defined on four components vector-valued functions (wavefunctions) $\psi=\left(\psi_{1}, \ldots, \psi_{4}\right)^{T} ; V=V(x)$ (the potential) describes the external field, that in fact represents an operator of multiplication with a $4 \times 4$ matrix-valued function of the space-time variable $x=\left(x_{1}, x_{2}, x_{3}\right) \in \mathbb{R}^{3}$.

In case the potential $V(x)$ is spherically symmetric, that is, it depends only on the distance between the Dirac particle and origin, the Dirac operator admits a unique, up to an unitary equivalence, representation in a direct sum (partial-wave decomposition) of one-dimensional Dirac operators of the form

$$
H=i^{-1} c \sigma_{2} \partial_{x}+\sigma_{3} m c^{2}+V,
$$


where $V$ is now an operator of multiplication by a $2 \times 2$ matrix-valued measurable function $V(x)$ defined on the half-line $\mathbb{R}_{+}:=[0, \infty)$.

The paper in question is concerned with the operator $H$ given formally by (4.2). The operator $H$ is considered to be defined on the space of vector-valued function $L_{2}\left(\mathbb{R}_{+}\right) \otimes \mathbb{C}^{2}=L_{2}\left(\mathbb{R}_{+}\right) \oplus L_{2}\left(\mathbb{R}_{+}\right)=L_{2}\left(\mathbb{R}_{+}\right)^{2}$. We assume for simplicity that $V$ satisfies conditions, such as locally summability and, in a certain average sense, to be vanishing at infinity, in order to ensure the relatively compactness of the operator $V$ with respect to the free Dirac operator

$$
H_{0}=i^{-1} c \sigma_{2} \partial_{x}+\sigma_{3} m c^{2} .
$$

Then the Dirac operator $H$ is well defined on the same domain as $H_{0}$ that is natural to take the Sobolev space $W_{2}^{1}\left(\mathbb{R}_{+}\right) \oplus \stackrel{\circ}{W_{2}^{1}}\left(\mathbb{R}_{+}\right)$of all vector-valued functions $u=$ $\left(u_{1}, u_{2}\right)^{\top}$ in $L_{2}\left(\mathbb{R}_{+}\right) \oplus L_{2}\left(\mathbb{R}_{+}\right)$whose components $u_{j}(j=1,2)$ are representing locally absolutely continuous functions with derivatives belonging to $L_{2}\left(\mathbb{R}_{+}\right)$and $u_{2}(0)=0$ (a Dirichlet boundary condition). Under these conditions $H$ represents a closed operator, and, in particular, a self-adjoint operator whenever $V(x)$ is Hermitian, having the same essential spectrum as the free Dirac operator $H_{0}$, that is,

$$
\sigma_{e s s}(H)=\left(-\infty,-m c^{2}\right] \cup\left[m c^{2}, \infty\right) .
$$

Outside of the essential spectrum, due to Weyl type theorem, there can be a nontrivial discrete part of the spectrum of $H$. By the discrete spectrum of $H$ we mean the set of isolated eigenvalues each of which being of finite multiplicity. The accumulation points of this set of eigenvalues can be only on the essential spectrum. We evaluate these eigenvalues, derive bounds on their location and describe the behaviour of them, especially, in self-adjoint case, in the spectral gap created in the essential spectrum. For the massless Dirac operators, whose essential spectrum is the entire real axis, we establish bounds for the imaginary part of the eigenvalues. The solutions of these problems, as shown in the paper, depend essentially on the asymptotic behaviour of the resolvent near the continuous spectrum and also at infinity. In this context, we analyse at first the asymptotic behaviour of the resolvent of the Dirac operator $H_{0}$ and, then, derive the desired estimates for eigenvalues.

The resolvent $R\left(H_{0} ; \lambda\right)$ of $H_{0}$ is an integral operator the kernel of which is given by a matrix-valued function being a smooth enough function and decreasing rapidly at infinity. Due to these properties, the resolvent $R\left(H_{0} ; \lambda\right)$ can be regarded as a bounded operator acting from $L_{p}\left(\mathbb{R}_{+}\right)^{2}$ into $L_{q}\left(\mathbb{R}_{+}\right)^{2}$ with some appropriate $p, q, 1 \leq p, q \leq \infty$. In fact, we prove the following

$$
\left\|R\left(H_{0} ; \lambda\right)\right\|_{L_{p} \rightarrow L_{q}} \leq c^{-1 / \alpha^{\prime}}(\alpha \operatorname{Im} \mu)^{-1 / \alpha} M(\lambda),
$$

provided that $1 \leq p \leq q \leq \infty$ and $p^{-1}-q^{-1}<1$, where

$$
\begin{gathered}
M(\lambda):=\max \left\{\frac{\left|\lambda+m c^{2}\right|}{|\mu|}, \frac{\left|\lambda-m c^{2}\right|}{|\mu|}\right\}+1, \\
\alpha:=\left(1-p^{-1}+q^{-1}\right)^{-1}, \alpha^{\prime}=\alpha(\alpha-1)^{-1}, \mu=\left(\lambda^{2}-m^{2} c^{4}\right)^{1 / 2} \text { with } \operatorname{Im} \mu>0 .
\end{gathered}
$$


We show also that the resolvent $R\left(H_{0} ; \lambda\right)$ is bounded as an operator acting from $L_{1}\left(\mathbb{R}_{+}\right)^{2}$ into $L_{\infty}\left(\mathbb{R}_{+}\right)^{2}(p=1$ and $q=\infty)$. For this case we have

$$
\left\|R\left(H_{0} ; \lambda\right)\right\|_{L_{1} \rightarrow L_{\infty}} \leq \frac{M(\lambda)}{c},
$$

where $M(\lambda)$ is determined as in (4.4).

Based on evaluations effectuated for the resolvent of the operator $H_{0}$ we obtain estimates for the perturbed eigenvalues. The methods we apply are in a proper agreement with the technique of factoring the perturbation described in Section 1.2. To this end we assume that the matrix $V(x)$ admits a factorization

$$
V(x)=A(x) B(x), \quad x \in \mathbb{R}_{+} \text {a.e. },
$$

with

$$
|A(x)| \in L_{r}\left(\mathbb{R}_{+}\right), \quad|B(x)| \in L_{s}\left(\mathbb{R}_{+}\right)
$$

for some $0<r, s \leq \infty$. Besides,

$$
|B(x)| \in L_{p, l o c}\left(\mathbb{R}_{+}\right), \quad \sup _{x>0} \int_{|y-x|<1}|B(y)|^{p} d y<\infty
$$

for some $2 \leq p<\infty$.

From (4.4) it is obtained that the perturbed eigenvalues lying outside the essential spectrum of the Dirac operator $H$ are confined in a region determined by

$$
(\alpha \operatorname{Im} \mu)^{1 / \alpha} \leq M(\lambda) c^{-1 / \alpha^{\prime}}\|A\|_{r}\|B\|_{s}
$$

provided that $0<r \leq \infty, 2 \leq p \leq s \leq \infty, r^{-1}+p^{-1} \leq 1, r^{-1}+s^{-1}<1$, where $M(\lambda)$ is as in (4.4), and $\alpha=\left(1-r^{-1}-s^{-1}\right)^{-1}$. Here $\|A\|_{r}$ (resp. $\left.\|B\|_{s}\right)$ denotes $L_{r}$-norm (resp. $L_{s}$-norm) of the function $|A(\cdot)|$ (resp. $\left.|B(\cdot)|\right)$.

For the limiting case when $\alpha=\infty$, that is, $r^{-1}+s^{-1}=1$, one has $s=p \geq 2$, $r=p^{\prime}$, and, the perturbed eigenvalues are confined in the region determined by

$$
c \leq M(\lambda)\|A\|_{p^{\prime}}\|B\|_{p} .
$$

From the estimates (4.6) and (4.7) one can infer information on the behaviour of the eigenvalues at infinity, near the points of the continuous spectrum, in particular, at $\pm m c^{2}$. For the self-adjoint case, when the discrete spectrum can be created only in the spectral gap $\left(-m c^{2}, m c^{2}\right)$ the estimates (4.6) and (4.7) can easily modify by taking into account that, in this case, $\operatorname{Im} \mu=\left(m^{2} c^{4}-\lambda^{2}\right)^{1 / 2}$. So, from (4.6), it is obtained

$$
\left(m c^{2}-\lambda\right)^{1-\alpha}\left(m c^{2}+\lambda\right)^{1+\alpha} \leq C\|A\|_{r}^{2 \alpha}\|B\|_{s}^{2 \alpha} \text { for } \lambda \leq 0,
$$

and

$$
\left(m c^{2}-\lambda\right)^{1+\alpha}\left(m c^{2}+\lambda\right)^{1-\alpha} \leq C\|A\|_{r}^{2 \alpha}\|B\|_{s}^{2 \alpha} \text { for } \lambda \geq 0
$$

with the same constant $C=2^{2 \alpha} \alpha^{-2} c^{2(1-\alpha)}$. By (4.8) one can get that the eigenvalues (belonging to the spectral gap) are contained in certain intervals $\left(-m c^{2},-a\right)$ and $\left(a, m c^{2}\right)$ separated by zero with

$$
a=m c^{2}(1-b)(1+b)^{-1}, \quad b=4 c^{-2}\|A\|_{p^{\prime}}^{2}\|B\|_{p}^{2},
$$


if, in addition,

$$
\|A\|_{p^{\prime}}\|B\|_{p} \leq c / 2 .
$$

In the general case when the potential $V$ is not necessarily Hermitian the condition (4.8) assures that the discrete spectrum of the perturbed Dirac operator $H$ is contained to the union of two disks centered at the points $\pm m c^{2}\left(1+b^{2}\right)\left(1-b^{2}\right)^{-1}$ and having the same radius equals to $2 m c^{2} b\left(1-b^{2}\right)^{-1}$. Such discs are discussed in [CLT14] for the case of the Dirac operator considered on the whole real axis assuming that the potential is a Lebesgue summable function with sufficiently small norm.

Next, we consider the questions raised in discussion for the massless Dirac operator (when the mass of the particle is neglected). It is of the form

$$
H=i^{-1} c \sigma_{2} \partial_{x}+V
$$

The behaviour of the massless Dirac operator differs fundamentally from the familiar cases of Dirac operators in which the mass is considered (i.e., $m \neq 0$ ). The spectrum of the free operator $H_{0}\left(=i^{-1} c \sigma_{2} \partial_{x}\right)$ covers the whole real axis and is purely absolutely continuous. In this case, in the evaluation of the resolvent $R\left(H_{0} ; \lambda\right)$, the factor $M(\lambda)$ becomes equal to 2 since $\mu=\lambda$ if $\operatorname{Im} \lambda>0$ and $\mu=-\lambda$ if $\operatorname{Im} \lambda<0$. Thus, in comparison with (4.4), we have

$$
\left\|R\left(H_{0} ; \lambda\right)\right\|_{L_{p} \rightarrow L_{q}} \leq 2 c^{-1 / \alpha^{\prime}} \alpha^{-1 / \alpha}|\operatorname{Im} \lambda|^{-1 / \alpha} .
$$

In the limiting case when $\alpha=\infty$ (the case in which $p=1$ and $q=\infty$ ), we have only

$$
\left\|R\left(H_{0} ; \lambda\right)\right\|_{L_{1} \rightarrow L_{\infty}} \leq 2 c^{-1} .
$$

Corresponding, for the perturbed eigenvalues outside the essential spectrum (which now consists of the whole real axis) satisfy

$$
|\operatorname{Im} \lambda| \leq C\|A\|_{r}\|B\|_{s},
$$

where $C=2 \alpha^{-1 / \alpha} c^{-1 / \alpha^{\prime}}$, provided that $0<r<\infty, p \leq s \leq \infty, r^{-1}+p^{-1} \leq 1$ and $r^{-1}+s^{-1}<1$. In the limiting case, where $r=p^{\prime}$ and $s=p$, it follows only an inequality like (4.8). In particular, if

$$
\int_{0}^{\infty}|V(x)|<c / 2,
$$

then $\lambda$ cannot be an eigenvalue of $H$ and, consequently, under last condition the massless Dirac operator has not any complex eigenvalues. A similar remark was pointed out in [CLT14] for the 1D Dirac operator considered on the real line assuming that the potentials are sufficiently small in $L_{1}$-norm.

Various estimates useful in applications such as those involving slowly decaying potentials can be derived directly from the general results. Thus, for instance, if it is taken

$$
A(x)=(1+x)^{-\tau} I_{2} \text { and } B(x)=(1+x)^{\tau} V(x)
$$


with an appropriate $\tau>0$, then for any complex eigenvalue of the massless Dirac operator $H$ there holds

$$
|\operatorname{Im} \lambda|^{r-2} \leq C \int_{0}^{\infty}\left|(1+x)^{\tau} V(x)\right|^{r} d x
$$

under restrictions $r \geq p \geq 2, r \neq 2, \tau r>1$, and with $C=2^{r} c^{-2}\left(1-2 r^{-1}\right)^{r-2}(\tau r-$ $1)^{-1}$.

Note that similar estimates are valid involving other weight functions as, for instance, $e^{\tau x}, e^{\tau x^{2}}, x^{\alpha} e^{\tau x^{\beta}}$, etc.

The results considered admit extensions to the multidimensional case of Dirac operators and also to more general situations in which the potentials are assumed to be of the weak Lebesgue classes. For the arguments used in [Enb17] and [Enb16] used for related problems concerning Schrödinger operators can be applied. 



\section{5 - Discussion of Paper IV}

\subsection{Overview of Paper IV}

This paper deals with Hardy-Carleman type inequalities for Dirac operators. By a Dirac operator we mean a first-order matrix differential operator of the form

$$
H=\sum_{j=1}^{n} \alpha_{j} D_{j}+\beta
$$

where $D_{j}=-i \partial / \partial x_{j} \quad(j=1, \ldots, n), x=\left(x_{1}, \ldots, x_{n}\right) \in \mathbb{R}^{n}, \alpha_{j}(j=1, \ldots, n)$ and $\beta$ are $m \times m$ constant Hermitian matrices which satisfy the Clifford's anticommutation relations

$\alpha_{j} \alpha_{k}+\alpha_{k} \alpha_{j}=2 \delta_{j k} I_{m}(j, k=1, \ldots, n), \quad \alpha_{j} \beta+\beta \alpha_{j}=0(j=1, \ldots, n), \quad \beta^{2}=I_{m}$, $m=2^{n / 2}$ for $n$ even and $m=2^{(n+1) / 2}$ for $n$ odd $\left(I_{m}\right.$ is the unit $m \times m$ matrix, and $\delta_{j k}$ is Kronecker's delta, i.e., $\delta_{j k}=1$ for $j=k$ and $\delta_{j k}=0$ for $j \neq k$ ). The Dirac operator $H$ is usually considered to be acting in the Hilbert space $L_{2}\left(\mathbb{R}^{n} ; \mathbb{C}^{m}\right)$ $\left(=L_{2}\left(\mathbb{R}^{n}\right)^{m}\right)$ of all $\mathbb{C}^{m}$-valued functions square Lebesgue summable functions and taken with domain the Sobolev space $W_{2}^{1}\left(\mathbb{R}^{n} ; \mathbb{C}^{m}\right)$. Nevertheless, in order to cover the most general situations, important for applications or also by themselves, we assume that the operator $H$ is given on the space $L_{2}\left(\Omega ; \mathbb{C}^{m}\right)$ of functions defined on an arbitrary (open) domain $\Omega . H$ is then taken with domain the Sobolev space $W_{2}^{1}\left(\Omega ; \mathbb{C}^{m}\right)$.

Our main purpose is to describe conditions for the weight functions $a$ and $b$ under which an inequality of the form

$$
c\|a u\| \leq\|b H u\|, \quad u \in \mathcal{D},
$$

holds true for a suitable class of functions $u \in \mathcal{D}, c$ being a positive constant depending only on $a$ and $b$, eventually, $\Omega$. For the sake of simplicity, we assume that $a$ and $b$ are sufficiently smooth functions, it will be enough to be of class $C^{2}$. In $(5.2),\|\cdot\|$ designates the norm of $L_{2}\left(\Omega ; \mathbb{C}^{m}\right)$. Estimates as $(5.2)$ we call Hardy-Carleman type inequalities. Inequalities of this kind, mainly related to the Laplace operator, are also called weighted Hardy inequalities or weighted PoincaréSobolev inequalities or weighted Friedrichs inequalities as well. We proceed from the classical Hardy inequalities for Dirichlet form (see, for instance, [KMP07] and [Maz85] and also [Dav99] for a history on the subject and further references), and the remarkable inequality due to Carleman stated in [Car39] in connection with the unique continuation property for second order elliptic differential equations. In addition to the works already mentioned, we refer to [ABG82], [ABG87] and [Hör83], [H0̈5], [Hör07], [Jer86], [JK85] and references cited therein. 
Despite the fact that the Laplace and Dirac operators are closely related to each other, they behave quite differently, and methods used for the Laplace operator do not apply to Dirac operators. In comparison to the case of the Laplace operator, the Hardy-Carleman inequalities regarding Dirac operators, have been studied rather sparingly in the literature. We note the paper [Jer86] in which certain Carleman type inequalities for the (massless) Dirac operator are established. In this context we remark the recent work [DES00] and also [DEDV07] and [DELV04], for some versions of Hardy inequalities related to the Dirac operator describing the behaviour of a spin $1 / 2$ particle with non-zero rest mass under the influence of an electrostatic potential $q$.

In what follows, we present the main results of the paper in question. We begin with the case of the Dirac operator considered on $\mathbb{R}^{n}$. It will be convenient to pass to polar coordinates. Recall that the polar coordinates $(r, \omega) \in(0, \infty) \times S^{n-1}$ in $\mathbb{R}^{n}$ are given by

$$
r=|x| \in(0, \infty), \quad \omega=x /|x| \in S^{n-1}
$$

for any $x \in \mathbb{R}^{n} \backslash\{0\}$. $S^{n-1}$ stands for the unit sphere in $\mathbb{R}^{n}$. Denoting $\omega_{j}=$ $x_{j} /|x|(j=1, \ldots, n)$, the coordinates of $\omega$, we have

$$
\frac{\partial}{\partial x_{j}}=\omega_{j} \frac{\partial}{\partial r}+\frac{1}{r} \Omega_{j} \quad(j=1, \ldots, n),
$$

where $\Omega_{j}$ is a vector-field on the unit sphere $S^{n-1}$ satisfying

$$
\sum_{j=1}^{n} \omega_{j} \Omega_{j}=0, \quad \sum_{j=1}^{n} \Omega_{j} \omega_{j}=r \sum_{j=1}^{n} \frac{\partial \omega_{j}}{\partial x_{j}}=n-1 .
$$

The Dirac operator given by (5.1) is written in polar coordinates as follows

$$
H=-i \widehat{\alpha} \frac{\partial}{\partial r}-i r^{-1} \sum_{j=1}^{n} \alpha_{j} \Omega_{j}+\beta,
$$

where

$$
\widehat{\alpha}=\sum_{j=1}^{n} \alpha_{j} \omega_{j}
$$

Passing to inequality (5.2), we set $v=b u, u \in \mathcal{D}$, and notice that

$$
b H b^{-1}=H+i B,
$$

where

$$
B=b^{-1} \sum_{j=1}^{n} \alpha_{j} \frac{\partial b}{\partial x_{j}}
$$

Taking this fact into account, an inequality of the form (5.2) reduces to estimation from below of the quadratic form

$$
h[v]=\|(H+i B) v\|^{2}
$$


on suitable elements $v$.

It turns out that if the matrix-valued function

$$
M(r, \omega)=r^{-1}\left(-i \widehat{\alpha} \beta+\widehat{\alpha} \frac{\partial(B(r \omega) r)}{\partial r}\right) b(r \omega)^{2} a(r \omega)^{-2}
$$

is positive definite (with respect to quadratic forms) uniformly with respect to $r$ and $\omega$, i.e., if

$$
\langle M(r, \omega) u(r \omega), u(r \omega)\rangle \geq c|u(r \omega)|^{2}
$$

for a positive constant $c$ independent of $u, r$ and $\omega$, then

$$
h[v] \geq c \int_{\mathbb{R}^{n}}|a(x) u(x)|^{2} d x,
$$

which leads to the desired inequality (5.2). For, as is seen, $\mathcal{D}$ can be taken as the set of all functions $u$ belonging to the Sobolev space $W_{2}^{1}\left(\mathbb{R}^{n} ; \mathbb{C}^{m}\right)$ having compact support in $\mathbb{R}^{n} \backslash\{0\}$.

In this way we get the following result.

Theorem 5.1.1. Let $a, b$ be (real) functions of the class $C^{2}$ for which (5.3) is satisfied. Then for any function $u$ belonging to the Sobolev space $W_{2}^{1}\left(\mathbb{R}^{n} ; \mathbb{C}^{m}\right)$ and having compact support in $\mathbb{R}^{n} \backslash\{0\}$ inequality (5.2) holds true with a positive constant $c$ depending only on $a$ and $b$.

This result is extended to the case of the Dirac operator considered on an arbitrary open domain $\Omega$. Then, the functions $u$ in (5.2) should be taken from the Sobolev space $W_{2}^{1}\left(\Omega ; \mathbb{C}^{m}\right)$ that have compact support in $\Omega$, respectively, in $\Omega \backslash\{0\}$ if $0 \in \Omega$. Clearly, condition (5.3) must be met on $\Omega$. On the other hand, condition (5.3) can be interpreted as defining domain $\Omega$ so that inequality (5.2) is held.

Special cases which occur in various situations in applications may be derived directly from general inequality (5.2). Thus, if

$$
a(x)=|x|^{-1 / 2} e^{\tau \varphi(x)}, \quad b(x)=e^{\tau \varphi(x)}, x \neq 0,
$$

with $\tau>0$ as a parameter and $\varphi$, being a function of the class $C^{2}$, depends only on the radial coordinate $r$, then for any function $u$ in the Sobolev space $W_{2}^{1}\left(\Omega ; \mathbb{C}^{m}\right)$ having compact support in $\Omega \backslash\{0\}$, the following Carleman inequality

$$
c \int_{\Omega}|x|^{-1} e^{2 \tau \varphi(x)}|u(x)|^{2} d x \leq \int_{\Omega} e^{2 \tau \varphi(x)}|H u(x)|^{2} d x
$$

holds true for sufficiently large $\tau$ and a positive constant $c$ depending only on $\tau$ and $\varphi$.

Examples of Hardy-Carleman type inequalities can be obtained depending on the choice of the weight functions $a, b$. With the aim of extending those inequalities that are well-known for the Laplace operator we restrict ourselves to the Dirac operator describing a relativistic particle with negligible mass, that is, the massless Dirac operator. Denote it by $H_{0}$, so (the term containing $\beta$ is now absent)

$$
H_{0}=\sum_{j=1}^{n} \alpha_{j} D_{j}
$$


of course, with all attributed conditions as in general case.

The cases considered involve weight functions depending only on the radial coordinate $r$. In turn the matrix-valued function $M(r, \omega)$ reduces to a scalar function (denote it by $M(r)$ ) depending only on $r$, namely,

$$
M(r)=r^{-1}\left(b(r)^{-1} b^{\prime}(r) r\right)^{\prime} b(r)^{2} a(r)^{-2} .
$$

Now, setting

$$
a(x)=\left(1+|x|^{2}\right)^{(\tau-2) / 2}, \quad b(x)=\left(1+|x|^{2}\right)^{\tau / 2}
$$

with $\tau>0$, we obtain that

$$
M(r)=2 \tau>0,
$$

and thus we have the following inequality

$$
2 \tau \int_{\mathbb{R}^{n}}\left(1+|x|^{2}\right)^{\tau-2}|u(x)|^{2} d x \leq \int_{\mathbb{R}^{n}}\left(1+|x|^{2 \tau}\right)\left|H_{0} u(x)\right|^{2} d x,
$$

for all $\tau>0$.

In (5.4) and all subsequent inequalities as well, the involving functions $u$ are assumed belonging to the Sobolev space $W_{2}^{1}\left(\mathbb{R}^{n} ; \mathbb{C}^{m}\right)$ and having compact support in the set $\mathbb{R}^{n} \backslash\{0\}$.

If in (5.4) it is taken $\tau=1$ or $\tau=2$, then

$$
2 \int_{\mathbb{R}^{n}}\left(1+|x|^{2}\right)^{-1}|u(x)|^{2} d x \leq \int_{\mathbb{R}^{n}}\left(1+|x|^{2}\right)\left|H_{0} u(x)\right|^{2} d x
$$

or, respectively,

$$
4 \int_{\mathbb{R}^{n}}|u(x)|^{2} d x \leq \int_{\mathbb{R}^{n}}\left(1+|x|^{2}\right)^{2}\left|H_{0} u(x)\right|^{2} d x .
$$

Inequality (5.5) can be viewed as an Agmon type inequality (see [Agm75] for a related inequality that concerns the Laplace operator), whereas (5.6) as a Hardy inequality for the Dirac operator. So, if

In the next example as weights are taken the functions of the form $|x|^{\sigma} e^{\tau|x|^{2}}$.

$$
a(x)=|x|^{(\alpha-2) / 2} e^{\tau|x|^{\alpha} / 2}, \quad b(x)=e^{\tau|x|^{\alpha} / 2}
$$

with $\tau>0, \alpha \in \mathbb{R}, \alpha \neq 0$, then

$$
M(r)=\alpha^{2} r / 2>0
$$

and, consequently, the following inequality is obtained

$$
\left(\alpha^{2} \tau / 2\right) \int_{\mathbb{R}^{n}}|x|^{\alpha-2} e^{\tau|x|^{\alpha}}|u(x)|^{2} d x \leq \int_{\mathbb{R}^{n}} e^{\tau|x|^{\alpha}}\left|H_{0} u(x)\right|^{2} d x .
$$

Particular cases of (5.7) are the following useful inequalities

$$
(\tau / 2) \int_{\mathbb{R}^{n}}|x|^{-1} e^{\tau|x|}|u(x)|^{2} d x \leq \int_{\mathbb{R}^{n}} e^{\tau|x|}\left|H_{0} u(x)\right|^{2} d x,
$$


and

$$
2 \tau \int_{\mathbb{R}^{n}} e^{\tau|x|^{2}}|u(x)|^{2} d x \leq \int_{\mathbb{R}^{n}} e^{\tau|x|^{2}}\left|H_{0} u(x)\right|^{2} d x,
$$

corresponding to the values $\alpha=1$ and $\alpha=2$, respectively. By analogy with known inequalities regarding elliptic differential operators [Tre61], (5.9) can be termed as a Treve type inequality for the Dirac operator $H_{0}$. It is worth mentioning that the constants in (5.7), (5.8), (5.9) as well as (5.4), (5.5) and (5.6) are optimal.

Finally, let

$$
a(x)=|x|^{-1} e^{\tau(\log |x|)^{2} / 2}, \quad b(x)=e^{\tau(\log |x|)^{2} / 2}
$$

for $x \neq 0$ and $\tau>0$. In this case

$$
M(r)=\tau>0,
$$

and we have

$$
\tau \int_{\mathbb{R}^{n}}|x|^{-2} e^{\tau(\log |x|)^{2}}|u(x)|^{2} d x \leq \int_{\mathbb{R}^{n}} e^{\tau(\log |x|)^{2}}\left|H_{0} u(x)\right|^{2} d x .
$$

Such an inequality is stated in [Jer86], however, instead of the whole space $\mathbb{R}^{n}$ it is taken the domain $\Omega$ determined by $a<|x|<b$ with $0<a<b<1$. In [Jer86] it is in fact proved the following inequality

$$
\left\|e^{\tau \varphi} u\right\|_{L^{q}\left(\Omega ; \mathbb{C}^{m}\right)} \leq C\left\|e^{\tau \varphi} H_{0} u\right\|_{L^{2}\left(\Omega ; \mathbb{C}^{m}\right)} \text { for all } u \in C_{0}^{\infty}\left(\Omega ; \mathbb{C}^{m}\right),
$$

where $\varphi(x)=(\log |x|)^{2} / 2, \tau>0, q=(6 n-4) /(3 n-6)$, and $C$ depending only on $a, b$ and $n$.

A special situation occurs with the inequality

$$
c \int_{\mathbb{R}^{n}}|x|^{\tau}|u(x)|^{2} d x \leq \int_{\mathbb{R}^{n}}|x|^{\tau+2}\left|H_{0} u(x)\right|^{2} d x, \quad \tau \in \mathbb{R},
$$

in which the weight functions are

$$
a(x)=|x|^{\tau / 2}, \quad b(x)=|x|^{(\tau+2) / 2} .
$$

It is easily seen that $M(r)$ is identically equal to zero in this case and the general results presented above cannot be applied. The following is however true.

Theorem 5.1.2. Let $n>1$ and let $\tau$ be a real number such that $\tau \neq 2 k-n$ for $k \in \mathbb{Z}$. The inequality (5.11) holds true for any function $u$ in the Sobolev space $W_{2}^{1}\left(\mathbb{R}^{n} ; \mathbb{C}^{m}\right)$ having compact support in the set $\mathbb{R}^{n} \backslash\{0\}$, where $c$ is a positive constant depending only on $d:=\min _{k \in \mathbb{Z}}|\tau+n-2 k|$.

The arguments in the proof of Theorem 5.1.2 use the eigenfunctions expansion with respect to spherical harmonic functions.

The methods used can be applied to more general cases like the Dirac operator with a magnetic field. Such an operator is defined by

$$
H_{A}=\sum_{j=1}^{n} \alpha_{j}\left(D_{j}-A_{j}(x)\right)+\beta,
$$


where $A(x)=\left(A_{1}(x), \ldots, A_{n}(x)\right)$ is a vector potential describing the magnetic field.

Assuming that the magnetic field $A$ has the form

$$
A=\nabla \varphi
$$

where $\varphi$ is a real-valued function with some prescribed (smoothness) properties $\left(\nabla=\left(\partial / \partial x_{1}, \ldots, \partial / \partial x_{n}\right)\right.$ denotes the gradient operator $)$, weighted estimates like those discussed above can be established as well.

\section{References}

[AAD01] A. A. Abramov, A. Aslanyan, and E. B. Davies. Bounds on complex eigenvalues and resonances. J. Phys. A, 34(1):57-72, 2001.

[ABG82] W. Amrein, A. Berthier, and V. Georgescu. Estimations du type Hardy ou Carleman pour des opérateurs différentiels à coefficients opératoriels. C. R. Acad. Sci. Paris Sér. I Math., 295(10):575-578, 1982.

[ABG87] W. Amrein, A. Berthier, and V. Georgescu. Hardy type inequalities for abstract differential operators. Mem. Amer. Math. Soc., $70(375): \mathrm{iv}+119,1987$.

[Agm75] S. Agmon. Spectral properties of Schrödinger operators and scattering theory. Ann. Scuola Norm. Sup. Pisa Cl. Sci. (4), 2(2):151-218, 1975.

[BL76] J. Bergh and J. Löfström. Interpolation spaces. An introduction. Springer-Verlag, Berlin, 1976. Grundlehren der Mathematischen Wissenschaften, No. 223.

[BS87] M. Sh. Birman and M. Z. Solomjak. Spectral theory of selfadjoint operators in Hilbert space. Mathematics and its Applications (Soviet Series). D. Reidel Publishing Co., Dordrecht, 1987. Translated from the 1980 Russian original by S. Khrushchëv and V. Peller.

[BS91] F. A. Berezin and M. A. Shubin. The Schrödinger equation, volume 66 of Mathematics and its Applications (Soviet Series). Kluwer Academic Publishers Group, Dordrecht, 1991.

[Car39] T. Carleman. Sur un problème d'unicité pur les systèmes d'équations aux dérivées partielles à deux variables indépendantes. Ark. Mat., Astr. Fys., 26(17):9, 1939.

[CLT14] J.-C. Cuenin, A. Laptev, and C. Tretter. Eigenvalue estimates for non-selfadjoint Dirac operators on the real line. Ann. Henri Poincaré, 15(4):707-736, 2014.

[Dav89] E. B. Davies. Heat kernels and spectral theory, volume 92 of Cambridge Tracts in Mathematics. Cambridge University Press, Cambridge, 1989. 
[Dav99] E. B. Davies. A review of Hardy inequalities. In The Maz' ya anniversary collection, Vol. 2 (Rostock, 1998), volume 110 of Oper. Theory Adv. Appl., pages 55-67. Birkhäuser, Basel, 1999.

[Dav02] E. B. Davies. Non-self-adjoint differential operators. Bull. London Math. Soc., 34(5):513-532, 2002.

[DEDV07] J. Dolbeault, M. J. Esteban, J. Duoandikoetxea, and L. Vega. Hardytype estimates for Dirac operators. Ann. Sci. École Norm. Sup. (4), 40(6):885-900, 2007.

[DELV04] J. Dolbeault, M. J. Esteban, M. Loss, and L. Vega. An analytical proof of Hardy-like inequalities related to the Dirac operator. J. Funct. Anal., 216(1):1-21, 2004.

[DES00] J. Dolbeault, M. J. Esteban, and E. Séré. On the eigenvalues of operators with gaps. Application to Dirac operators. J. Funct. Anal., 174(1):208-226, 2000.

[DN02] E. B. Davies and Jiban Nath. Schrödinger operators with slowly decaying potentials. J. Comput. Appl. Math., 148(1):1-28, 2002.

[DS88] N. Dunford and J. T. Schwartz. Linear operators. Part I. Wiley Classics Library. John Wiley \& Sons, Inc., New York, 1988. General theory, With the assistance of William G. Bade and Robert G. Bartle, Reprint of the 1958 original, A Wiley-Interscience Publication.

[Enb16] A. Enblom. Estimates for eigenvalues of Schrödinger operators with complex-valued potentials. Lett. Math. Phys., 106(2):197-220, 2016.

[Enb17] A. Enblom. Estimates of eigenvalues of Schrödinger operators on the half-line with complex-valued potentials. Oper. Matrices, 11(2):369$380,2017$.

[FH10] S. Fournais and B. Helffer. Spectral methods in surface superconductivity, volume 77 of Progress in Nonlinear Differential Equations and their Applications. Birkhäuser Boston, Inc., Boston, MA, 2010.

[FLS11] R. L. Frank, A. Laptev, and R. Seiringer. A sharp bound on eigenvalues of Schrödinger operators on the half-line with complex-valued potentials. In Spectral theory and analysis, volume 214 of Oper. Theory Adv. Appl., pages 39-44. Birkhäuser/Springer Basel AG, Basel, 2011.

[Foc78] V. Fock. Fundamentals of Quantum Mechanics. MIR Publishers Moscow, 1978.

[Fra11] R. L. Frank. Eigenvalue bounds for Schrödinger operators with complex potentials. Bull. Lond. Math. Soc., 43(4):745-750, 2011. 
[GKn69] I. C. Gohberg and M. G. Kreй n. Introduction to the theory of linear non-self-adjoint operators. Translated from the Russian by A. Feinstein. Translations of Mathematical Monographs, Vol. 18. American Mathematical Society, Providence, R.I., 1969.

[Gla66] I. M. Glazman. Direct methods of qualitative spectral analysis of singular differential operators. Translated from the Russian by the IPST staff. Israel Program for Scientific Translations, Jerusalem, 1965; Daniel Davey \& Co., Inc., New York, 1966.

[Goh51] I. C. Gohberg. On linear operators depending analytically on a parameter. Doklady Akad. Nauk SSSR (N.S.), 78:629-632, 1951.

[GR07] I. S. Gradshteyn and I. M. Ryzhik. Table of integrals, series, and products. Elsevier/Academic Press, Amsterdam, seventh edition, 2007.

[Gru09] G. Grubb. Distributions and operators, volume 252 of Graduate Texts in Mathematics. Springer, New York, 2009.

[H0̈5] L. Hörmander. The analysis of linear partial differential operators. II. Classics in Mathematics. Springer-Verlag, Berlin, 2005. Differential operators with constant coefficients, Reprint of the 1983 original.

[Hör83] L. Hörmander. Uniqueness theorems for second order elliptic differential equations. Comm. Partial Differential Equations, 8(1):21-64, 1983.

[Hör07] L. Hörmander. The analysis of linear partial differential operators. III. Springer, Berlin, 2007. Pseudo-differential operators.

[HP74] E. Hille and R. S. Phillips. Functional analysis and semi-groups. American Mathematical Society, Providence, R. I., 1974.

[Jer86] D. Jerison. Carleman inequalities for the Dirac and Laplace operators and unique continuation. Adv. in Math., 62(2):118-134, 1986.

[JK85] D. Jerison and C. E. Kenig. Unique continuation and absence of positive eigenvalues for Schrödinger operators. Ann. of Math. (2), 121(3):463-494, 1985.

[JW73] K. Jörgens and J. Weidmann. Spectral properties of Hamiltonian operators. Lecture Notes in Mathematics, Vol. 313. Springer-Verlag, Berlin, 1973.

[Kat66] T. Kato. Wave operators and similarity for some non-selfadjoint operators. Math. Ann., 162:258-279, 1965/1966.

[Kat95] T. Kato. Perturbation theory for linear operators. Classics in Mathematics. Springer-Verlag, Berlin, 1995. Reprint of the 1980 edition. 
[Kel61] J. B. Keller. Lower bounds and isoperimetric inequalities for eigenvalues of the Schrödinger equation. J. Mathematical Phys., 2:262-266, 1961.

[KK66] R. Konno and S. T. Kuroda. On the finiteness of perturbed eigenvalues. J. Fac. Sci. Univ. Tokyo Sect. I, 13:55-63 (1966), 1966.

[KMP07] A. Kufner, L. Maligranda, and L.-E. Persson. The Hardy inequality. About its history and some related results. Vydavatelský Servis, Plzeň, 2007.

[KRS87] C. E. Kenig, A. Ruiz, and C. D. Sogge. Uniform Sobolev inequalities and unique continuation for second order constant coefficient differential operators. Duke Math. J., 55(2):329-347, 1987.

[Kur67] S. T. Kuroda. An abstract stationary approach to perturbation of continuous spectra and scattering theory. J. Analyse Math., 20:57$117,1967$.

[Kur73a] S. T. Kuroda. Scattering theory for differential operators. I. Operator theory. J. Math. Soc. Japan, 25:75-104, 1973.

[Kur73b] S. T. Kuroda. Scattering theory for differential operators. II. Selfadjoint elliptic operators. J. Math. Soc. Japan, 25:222-234, 1973.

[LS91] B. M. Levitan and I. S. Sargsjan. Sturm-Liouville and Dirac operators, volume 59 of Mathematics and its Applications (Soviet Series). Kluwer Academic Publishers Group, Dordrecht, 1991. Translated from the Russian.

[LS09] A. Laptev and O. Safronov. Eigenvalue estimates for Schrödinger operators with complex potentials. Comm. Math. Phys., 292(1):29-54, 2009.

[LS10] E. H. Lieb and R. Seiringer. The stability of matter in quantum mechanics. Cambridge University Press, Cambridge, 2010.

[LT76] E. H. Lieb and W. E. Thirring. Inequalities for the moments of the eigenvalues of the Schrödinger Hamiltonian and their relation to Sobolev inequalities. In Studies in Mathematical Physics, pages 269303. Princeton Univ. Press, Princeton, NY, 1976.

[Maz85] V. G. Maz'ja. Sobolev spaces. Springer Series in Soviet Mathematics. Springer-Verlag, Berlin, 1985.

[O'N63] R. O'Neil. Convolution operators and $L(p, q)$ spaces. Duke Math. J., 30:129-142, 1963.

[Pro64] R. T. Prosser. Convergent perturbation expansions for certain wave operators. J. Mathematical Phys., 5:708-713, 1964. 
[RS72] M. Reed and B. Simon. Methods of modern mathematical physics. I. Functional analysis. Academic Press, New York-London, 1972.

[RS75] M. Reed and B. Simon. Methods of modern mathematical physics. II. Fourier analysis, self-adjointness. Academic Press [Harcourt Brace Jovanovich, Publishers], New York-London, 1975.

[RS78] M. Reed and B. Simon. Methods of modern mathematical physics. IV. Analysis of operators. Academic Press [Harcourt Brace Jovanovich, Publishers], New York-London, 1978.

[RS79] M. Reed and B. Simon. Methods of modern mathematical physics. III. Academic Press [Harcourt Brace Jovanovich, Publishers], New YorkLondon, 1979. Scattering theory.

[Saf10] O. Safronov. Estimates for eigenvalues of the Schrödinger operator with a complex potential. Bull. Lond. Math. Soc., 42(3):452-456, 2010.

[Sch60] J. Schwartz. Some non-selfadjoint operators. Comm. Pure Appl. Math., 13:609-639, 1960.

[Sch81] M. Schechter. Operator methods in quantum mechanics. North-Holland Publishing Co., New York-Amsterdam, 1981.

[Sch86] M. Schechter. Spectra of partial differential operators, volume 14 of North-Holland Series in Applied Mathematics and Mechanics. NorthHolland Publishing Co., Amsterdam, second edition, 1986.

[Ste69] S. Steinberg. Meromorphic families of compact operators. Arch. Rational Mech. Anal., 31:372-379, 1968/1969.

[Stu56] F. Stummel. Singuläre elliptische Differential-operatoren in Hilbertschen Räumen. Math. Ann., 132:150-176, 1956.

[SWW64] M. Scadron, S. Weinberg, and J. Wright. Functional analysis and scattering theory. Phys. Rev. (2), 135:B202-B207, 1964.

[Tam27] J. D. Tamarkin. On Fredholm's integral equations, whose kernels are analytic in a parameter. Ann. of Math. (2), 28(1-4):127-152, 1926/27.

[Tha92] B. Thaller. The Dirac equation. Texts and Monographs in Physics. Springer-Verlag, Berlin, 1992.

[Tre61] J. F Treves. Lectures on linear partial differential equations with constant coefficients. Notas de Matemátiea, No. 27. Instituto de Matemática Pura e Aplicada do Conselho Nacional de Pesquisas, Rio de Janeiro, 1961.

[Tri78] H. Triebel. Interpolation theory, function spaces, differential operators. VEB Deutscher Verlag der Wissenschaften, Berlin, 1978. 
[Wey09] H. Weyl. Uber beschränkte quadratische formen. Math. Ann., 27:373392, 1909.

[Wol52] F. Wolf. Analytic perturbation of operators in Banach spaces. Math. Ann., 124:317-333, 1952. 



\section{Papers}

The papers associated with this thesis have been removed for copyright reasons. For more details about these see:

http://urn.kb.se/resolve?urn=urn:nbn:se:liu:diva-145173 